\title{
Transcriptomic landscape of IncRNAs in inflammatory bowel disease
}

\author{
Aashiq H Mirza ${ }^{1,2,3 \dagger}$, Claus HB Berthelsen ${ }^{1,6+}$, Stefan E Seemann ${ }^{1,3}$, Xiaoyong Pan ${ }^{1,3,5}$, Klaus S Frederiksen", \\ Mogens Vilien ${ }^{4}$, Jan Gorodkin ${ }^{1,3}$ and Flemming Pociot ${ }^{1,2,3^{*}}$
}

\begin{abstract}
Background: Inflammatory bowel disease (IBD) is a complex multi-factorial inflammatory disease with Crohn's disease (CD) and ulcerative colitis (UC) being the two most common forms. A number of transcriptional profiling studies have provided compelling evidence that describe the role of protein-coding genes and microRNAs in modulating the immune responses in IBD.
\end{abstract}

Methods: In the present study, we performed a genome-wide transcriptome profiling of IncRNAs and protein-coding genes in 96 colon pinch biopsies (inflamed and non-inflamed) extracted from multiple colonic locations from 45 patients $(C D=13, U C=20$, controls $=12)$ using an expression microarray platform.

Results: In our study, we identified widespread dysregulation of IncRNAs and protein-coding genes in both inflamed and non-inflamed CD and UC compared to the healthy controls. In cases of inflamed CD and UC, we identified 438 and 745 differentially expressed IncRNAs, respectively, while in cases of the non-inflamed CD and UC, we identified 12 and 19 differentially expressed IncRNAs, respectively. We also observed significant enrichment ( $P$-value $<0.001$, Pearson's Chi-squared test) for 96 differentially expressed IncRNAs and 154 protein-coding genes within the IBD susceptibility loci. Furthermore, we found strong positive expression correlations for the intersecting and cis-neighboring differentially expressed IBD loci-associated IncRNA-protein-coding gene pairs. The functional annotation analysis of differentially expressed genes revealed their involvement in the immune response, pro-inflammatory cytokine activity and MHC protein complex.

Conclusions: The IncRNA expression profiling in both inflamed and non-inflamed CD and UC successfully stratified IBD patients from the healthy controls. Taken together, the identified IncRNA transcriptional signature along with clinically relevant parameters suggest their potential as biomarkers in IBD.

\section{Background}

Inflammatory bowel diseases (IBDs) are idiopathic chronic relapsing inflammatory conditions of the gastrointestinal tract. Crohn's disease (CD) and ulcerative colitis (UC) are two most common forms of the IBD. IBD is emerging as a global disease with its incidence and prevalence differentially increasing geographically around the world. Accumulating evidence suggests that IBDs result from the complex interplay between genetic, immunologic, and modifiable environmental factors [1]

\footnotetext{
* Correspondence: flemming.pociot.01@regionh.dk

${ }^{\dagger}$ Equal contributors

${ }^{1}$ Center for non-coding RNA in Technology and Health, University of Copenhagen, Frederiksberg 1870, Denmark

${ }^{2}$ Department of Pediatrics E, Copenhagen Diabetes Research Center (CPH-DIRECT), Herlev University Hospital, Herlev 2730, Denmark

Full list of author information is available at the end of the article
}

in a genetically susceptible host against a subset of gut commensal microbiota [2-4].

$\mathrm{CD}$ is characterized by intestinal inflammation in a discontinuous fashion and involves any part of the gastrointestinal tract, although in most cases the terminal ileum and/or colon is affected. A transmural pattern of inflammation is a hallmark of $\mathrm{CD}$ accompanied by other pathophysiological complications like thickened submucosa, intestinal fibrosis, fissuring ulceration in highly active disease, non-caseating granulomas, strictures, abscesses and fistulas [3]. By contrast, UC involves only the rectum and colon, and is characterized by superficial inflammation that is restricted to the mucosa and submucosa with the presence of cryptitis and crypt abscesses. Disease activity in both $\mathrm{CD}$ and $\mathrm{UC}$ is typically relapsing and remitting and both conditions are often difficult to diagnose 
because of idiosyncrasies in the presentation of overlapping and distinct clinical and pathological features [2,3]. Characteristically, diagnosis of either $\mathrm{CD}$ or $\mathrm{UC}$ is based on a number of findings, including clinical symptoms, endoscopic features, radiologic tests, and biopsy histology.

According to recent meta-analysis of IBD genomewide association studies data, the number of confirmed genetic loci associated with risk for IBD has increased to 163, with 110 shared between $\mathrm{CD}$ and UC, $30 \mathrm{CD}$ specific and 23 UC-specific. Interestingly, an overwhelming majority of these IBD loci are located in the noncoding intergenic and intronic regions [5]. Most overlap regulatory elements and consequently are likely to influence gene regulation. Findings from our recent studies have demonstrated that a large number of annotated long non-coding RNAs (lncRNAs), including novel evolutionarily conserved structured RNA motifs with regulatory potential [6] (SE Seemann et al., unpublished observations), overlap the IBD loci. Consistent with our findings, another recent study elegantly revealed that IBD loci overlap active regulatory regions in primary intestinal epithelium and immune cells and also were found significantly enriched within these active regulatory regions [7].

Several transcriptome profiling studies have provided compelling evidence describing the role of protein-coding and non-coding RNAs (ncRNAs), such as microRNAs, in modulating immune responses in IBD [8-15]. In murine models, loss of endogenous intestinal microRNAs is known to cause impairment of epithelial barrier function that results in acute inflammation [16]. Several studies have explored clinical differences between CD and UC based on transcriptional regulation [17,18]. Recently, Granlund et al. [9] demonstrated lack of major differences between $\mathrm{CD}$ and UC based on protein-coding gene expression profiling in IBD. In contrast, expression profiling of colon biopsies from IBD patients allude to differential diagnosis of $\mathrm{CD}$ and $\mathrm{UC}$ based on transcriptional signatures associated with intestinal inflammation [19].

LncRNAs have emerged as important regulators of gene expression, with an accumulating body of evidence linking lncRNAs to a plethora of human pathologies, including inflammatory diseases [20]. Although, the precise role of lncRNAs in intestinal diseases remains poorly understood, evidence from recent studies indicates that lncRNAs might be playing a crucial role in inflammatory cascades. Indeed, a preponderance of emerging evidence from a number of studies demonstrates important roles for lncRNAs in regulating gene expression within the immune system. Nevertheless, identification of IBD susceptibility loci has afforded limited success in translating results from gene expression studies to advance our knowledge and understanding of IBD pathophysiology. In particular, details about the initiation, propagation and maintenance of the lingering inflammation in IBD remains unclear. Furthermore, earlier transcriptomic studies in IBD have mostly focused on the protein-coding genes, with few profiling studies focusing on microRNAs. However, no study has explored the genome-wide expression profile of lncRNAs in IBD.

In the present study, transcriptomic profiling of lncRNAs and protein-coding genes from colon pinch biopsies of IBD patients was performed using an expression microarray platform. Our results identified widespread dysregulation of lncRNAs and protein-coding gene expression in both CD and UC. Notably, the differential transcriptomic signatures of lncRNAs and protein-coding genes in inflamed CD (iCD) and inflamed UC (iUC) enabled clear stratification of the $\mathrm{CD}$ and $\mathrm{UC}$ phenotypes. These data indicate that lncRNAs could potentially be used as predictive biomarkers in IBD.

\section{Methods}

\section{Sample collection for patients and controls}

All the patient samples were collected from an IBD cohort at North Zealand Hospital, Hillerød, Denmark. Subjects were required to meet the Copenhagen criteria for CD or UC. Participants recruited for the study were patients admitted to the Department of Gastroenterology for colonoscopy who were diagnosed either with $\mathrm{CD}$ or $\mathrm{UC}$, or were admitted to the clinic for diagnostic colonoscopy because of symptoms unrelated to the IBD. Written informed consent from all the participants in the study was acquired prior to the collection of samples and medical history. In total, 90 biopsies were collected from 45 individuals (13 CD, $20 \mathrm{UC}$ patients and 12 healthy controls). Subjects were included as normal controls only after all clinical examinations had concluded no signs of autoimmune or inflammatory disease. For the IBD groups ( $\mathrm{CD}$ and $\mathrm{UC}$ ), one to five endoscopic pinch biopsies were extracted from the macroscopically most inflamed mucosa (iCD/iUC) and adjacent non-inflamed (niCD/niUC) mucosa within colon (transverse, descending, sigmoid), ileum, transverse ileum and rectum for the $C D$ patients, and colon sigmoid and rectum for the UC patients. For the control group, one to five biopsies were taken from the same locations as in the CD group, except for two samples (G3_G1 and 60_G1) that were extracted from the duodenal bulb. All biopsies were placed in RNAlater solution (QIAGEN, Hilden, Germany), and stored for later downstream use. The study was approved by the Regional Ethical Committee (H-4-2012-030). The inflammation status of biopsies was confirmed by histologic examination and features of chronic intestinal inflammation for each patient were scored using a previously described scoring system for UC [21] and CD [22]. The pathologists were blinded to the status of inflammation. Additionally, we also tested expression of a panel of 26 pro-inflammatory 
markers (cytokines, interleukins, metalloproteases) using quantitative real-time PCR (qPCR; Fluidigm platform) to confirm the inflammation status of biopsies (data not shown) prior to the microarray analysis.

\section{RNA extraction and quality control}

Total RNA was extracted from biopsies stored in RNAlater using RNeasy Mini Kit (QIAGEN) according to the manufacturer's instructions. Briefly, the biopsy samples were homogenized in lysis buffer with $1.4 \mathrm{~mm}$ ceramic beads (MO BIO Laboratories, Carlsbad, CA, USA) using a Thermo Savant FastPrep FP120 Homogenizer (Carlsbad, CA, USA) for $30 \mathrm{~s}$ at a speed of $4 \mathrm{~m} / \mathrm{s}$. All the remaining steps of the protocol were performed according to the manufacturer's recommendations. To remove traces of genomic DNA, samples were treated with DNase I (QIAGEN). RNA was finally eluted with nuclease-free water supplied with the kit. The quantity and purity of isolated RNA was determined by UV absorbance using a NanoDrop 2000 Spectrophotometer (Thermo Scientific, Wilmington, DE, USA), and the integrity of RNA was assessed by analysis of rRNA band integrity on an Agilent 2100 Bioanalyzer RNA 6000 LabChip kit (Agilent Technologies). Only RNA samples with RNA integrity number (RIN) $>7$ were used for the microarray experiments.

\section{Microarray hybridization}

Total RNA (100 ng) was labeled using a LowInputQuick Amp Labeling kit v6.5 (Agilent 5190-2305) following the manufacturer's instructions. Briefly, mRNA was reverse transcribed in the presence of T7-oligo-dT primer to synthesize cDNA. The cDNA was then in vitro transcribed with T7 RNA polymerase in the presence of Cy3-CTP to generate labeled cRNA. The labeled cRNA was hybridized to the Agilent Custom $8 \times 60 \mathrm{~K}$ format lncRNA expression microarray (AMADID 047718, based on Gencode v.15 catalog of human long ncRNAs, probe length of 60 nucleotides) according to the manufacturer's protocol. Finally, the arrays were washed, and scanned on an Agilent G2565CA microarray scanner at 100\% PMT (photomultiplier tube) and $3 \mu \mathrm{m}$ resolution. Intensity data were extracted using the Feature Extraction software (Agilent). More detailed and general information about the array can also be found on the GENCODE website [23]. The raw microarray data reported in this manuscript have been deposited in the Gene Expression Omnibus (GEO) database with accession number GSE67106.

\section{Statistical analyses}

Raw data were corrected for background noise using the normexp method [24]. To assure comparability across samples, we used quantile normalization [25] (unpublished observations). Median intensity was taken between tech- nical replicates after checking pairwise Pearson correlation coefficients $\left(r^{2} \geq 0.98\right)$. Differential expression analysis was carried out on non-control probes with an empirical Bayes approach on linear models (LIMMA) [26]. Principal component analysis (PCA) was employed for the initial interpretation of the data. In total, we made seven comparisons to identify differentially expressed genes (iCD versus control, iUC versus control, iCD versus niCD, iUC versus niUC, niCD versus control, niUC versus control and $\mathrm{ICD}$ versus iUC) (Tables S1, S2, S3, and S4 in Additional file 1). $P$-values were adjusted for multiple comparisons using the false discovery rate (FDR) correction [27]. Differentially expressed genes were identified using the double-filtering criterion: adjusted $P$-value (FDR) $<0.05$ and an absolute fold change (absolute FC) $>1.5$. For transcripts targeted by two probes, only those probes that were changing in the same direction and the probes with highest FC values were retained for further analysis. All statistical analyses were performed with Bioconductor in the $\mathrm{R}$ statistical environment [28].

\section{Validation of differentially expressed genes by quantitative real-time PCR}

The expression of differentially expressed genes from microarray experiments was validated by qPCR using hydrolysis probe-based inventoried and custom designed PrimeTime qPCR 5' Nuclease assays procured from Integrated DNA Technologies, Coralville, IA, USA. The double-quenched hydrolysis probes with 5' FAM fluorophore, a 3' IBFQ quencher, and an internal $\mathrm{ZEN}^{\mathrm{m}}$ quencher were used for all assays. From the list of the top differentially expressed genes from the different comparisons, six up- and six down-regulated genes were selected for their expression validation by $\mathrm{qPCR}$ in a subset of samples used for the microarray experiments. Three upregulated (DUOXA2, CHI3L1, DST), and three downregulated (PCK1, KCNK10, and SERPINB3) protein-coding genes were validated, and three up-regulated ( $M M P 12$, RP11-731 F5.2, AC007182.6) and three down-regulated (DPP10-AS1, CDKN2B-AS1, and AL928742.12) lncRNA genes were validated. In addition, expression of the protein-coding gene $D U O X 2$ was also measured by qPCR. All cDNAs were prepared using 750 ng of DNA-free RNA using an iScript ${ }^{\mathrm{m} m \mathrm{c}} \mathrm{cDNA}$ synthesis kit (BioRad, Hercules, CA, USA) with a mixture of random and oligo(dT) primers following the manufacturer's instructions. Realtime PCR was performed with $7.5 \mathrm{ng}$ of cDNA per well template for all the protein-coding genes and lncRNAs with Brilliant III Ultra-Fast QPCR Master Mix (Agilent Technologies). For PCR amplification, the following thermal profile was used: 3 minutes at $95^{\circ} \mathrm{C} ; 40 \times\left(5 \mathrm{~s}\right.$ at $95^{\circ} \mathrm{C}$, $10 \mathrm{~s}$ at $60^{\circ} \mathrm{C}$ ). Expression of each lncRNA and proteincoding gene tested was represented as a FC using the $2^{-\triangle \triangle C T}$ method. GAPDH was used as the reference gene. 


\section{Identification of inflammatory bowel disease loci associated IncRNAs}

All IBD loci marker SNPs and associated genes were retrieved from ImmunoBase [29]. In total, 233 unique marker SNPs for IBD, CD, and UC regions were retrieved and mapped to the 22,007 lncRNAs (Gencode v.15) using the intersect feature of BedTools [30]. The susceptibility locus for IBD was defined based on a $500 \mathrm{~kb}$ long genomic region with the IBD marker SNP in the middle. The differentially expressed lncRNAs from five comparisons (iCD versus control, iUC versus control, $\mathrm{iCD}$ versus niCD, $\mathrm{iUC}$ versus niUC, and $\mathrm{iCD}$ versus $\mathrm{iUC}$ ) were mapped to the IBD loci to identify the IBD loci-associated lncRNAs. Regulatory evidence for the IBD-associated SNPs was retrieved from Mokry et al. [7] and RegulomeDB [31].

\section{Functional annotation and Gene Ontology analysis of differentially expressed IncRNAs}

For the differentially expressed lncRNAs, the nearest protein-coding neighbors within a span of $<10 \mathrm{~kb}$ were identified. For the antisense overlapping or intronic overlapping lncRNAs, intersecting protein-coding gene(s) were identified using the intersect feature of BedTools [30]. The PANTHER (protein annotation through evolutionary relationship) classification system [32] was used to perform functional annotation and Gene Ontology (GO) analysis of genes that overlap with or are neighbors of the differentially expressed lncRNAs. Likewise, for the IBD loci-associated lncRNAs, GO analysis was performed using the above described nearest neighbor approach. The enrichment for over-represented GO functional terms was calculated based on the binomial test in PANTHER.

\section{Sample classification using Support Vector Machines based on differentially expressed genes identified by LIMMA}

Support Vector Machines (SVM) [33] was used for classifying the $\mathrm{CD}$ and $\mathrm{UC}$ cases from the controls based on differentially expressed genes identified by LIMMA in five comparisons (iCD versus control, iCD versus niCD, iUC versus control, iUC versus niUC and iCD versus iUC). SVM classification was applied to all five comparisons using leave-one-out cross-validation for differentially expressed lncRNAs and protein-coding genes. To explore the effect of various clinical parameters (age, sex, smoking, disease index and biopsy location) on overall disease outcome, we used the following linear regression function:

$$
\begin{aligned}
y= & \text { err }+w 1^{*} \text { age }+w 2^{*} \operatorname{sex}+w 3^{*} \text { smoking } \\
& +w 4^{*} \text { disease index }+w 5^{*} \text { biopsy location }
\end{aligned}
$$

Here, $\mathrm{y}=1$ for the iCD or iUC disease phenotype and 0 for the rest of the samples. For the clinical parameters age and disease index we used original values, while for sex and smoking we used the following binary outcomes: male $=1$, female $=0$ and smoker $=1$, non-smoker $=0$. For the six biopsy locations, we used values ranging from 0 to 5 . To control any input bias, the same analysis was performed on a randomized lncRNA gene list with the same number of genes as the total differentially expressed lncRNA genes. The feature values were normalized to values ranging from 0 to 1 using ( $\mathrm{x}$ - Minimum)/ (Maximum - Minimum). Linear regression was applied using the Scikit-learn [34] package in Python and the least squares method was used for optimization in our analysis. Furthermore, differentially expressed genes identified by LIMMA were verified by the SVM-recursive feature elimination (SVM-RFE) method [35]. SVM-RFE recursively prunes genes whose absolute weights are the smallest until the desired number of features is reached. For each comparison, we used SVM-RFE to identify the same number of differentially expressed genes as identified by LIMMA.

\section{Co-expression network analysis}

To identify CD- and UC-specific network clusters (modules) based on highly correlated genes, the weighted correlation network analysis (WGCNA) method was used [36]. We used the normalized expression data as input and removed the outlier samples. The clinical parameters were represented as follows: numeric for age and disease, binary for sex, ethnicity (three categories), smoking (four categories), clinical subgroup (five categories), and biopsy location (six categories). The standard procedure of WGCNA was applied for network construction and module identification. The trait-based gene significance measure is defined as the absolute correlation and correlation test $P$-value between the trait and the gene expression profile. GO analysis of modules was performed with the GOstats package in $\mathrm{R}$ [37] using adjusted $P$-value $<0.001$. We controlled for study bias in the GO analysis by running the same analysis for randomized gene sets with the same module sizes.

\section{Results}

An overall summary of sample information is provided in Table 1. Both, CD and UC samples were divided based on inflammation status confirmed by macroscopic and microscopic evaluations and pro-inflammatory gene signatures into inflamed (iCD, iUC) and non-inflamed (niCD, niUC) categories. The total number of samples included $21 \mathrm{iCD}, 23 \mathrm{niCD}, 15 \mathrm{iUC}, 9$ niUC and 22 healthy controls. In total, 90 intestinal pinch biopsies (45 individuals (13 CD, 20 UC patients and 12 healthy controls)) from multiple colonic regions were harvested from both inflamed and non-inflamed mucosa (Figure 1A). Detailed sample information, including ethnic background, disease index, previous treatment regimens and other clinical parameters, are listed in Table 2. 
Table 1 Overall study design and sample information

\begin{tabular}{lll}
\hline Diagnosis & Number of samples & Number of individuals \\
\hline iCD & 21 & 13 \\
niCD & 23 & \\
iUC & 21 (15 unique) & 20 \\
niUC & 9 & \\
Controls & 22 & 12 \\
Total & 96 (90 unique samples) & 45
\end{tabular}

Ninety biopsy samples extracted from different colonic locations from 45 patients $(C D=13, U C=20$, controls $=12)$. Six samples from UC patients were used as technical replicates.

\section{Microarray analysis of IncRNAs and protein-coding gene expression}

In the Gencode v.15 lncRNA microarray design, each lncRNA transcript is targeted by two probes covering 22,001 lncRNA transcripts corresponding to 12,963 lncRNA genes. In addition, each array contains 17,535 randomly selected protein-coding targets, of which 15,182 (unique 12,787) correspond to protein-coding genes. Six samples analyzed in duplicate, hybridized on separate chips, and used as technical replicates showed strong positive Pearson correlation $\left(\mathrm{r}^{2} \geq 0.98, P\right.$-value $<2.2 \mathrm{e}-16$; Figure S1 in Additional file 2). Based on the PCA (see Methods section for details), separation of $\mathrm{iCD}$ and iUC samples from niCD, niUC and healthy controls were observed (Figure 1B). However, there was no apparent separation between iCD and iUC samples. The scatterplot matrices describing the first four principal components are described in Figure S2 in Additional file 2. Unsupervised hierarchical clustering of the most dynamic probes (coefficient of variance $>0.05$ ) across the samples resulted in clustering of samples according to their clinical subgroups (Figure 1C). The probes targeting lncRNAs and proteincoding genes separately also clustered samples in a similar manner (Figure S3A,B in Additional file 2).

\section{Differential transcriptional signature of IncRNAs and protein-coding genes in Crohn's disease and ulcerative colitis}

To define CD- and UC-specific transcriptional signatures based on intestinal inflammation, we identified differentially expressed genes using LIMMA [24] (based on a cutoff of $\log 2 \mathrm{FC}>1.5$ (up-regulated), $\mathrm{FC}<-1.5$ (down-regulated) and adjusted $P$-value $<0.05$ (moderated $t$-test)) in all comparisons (Table 3$)$. The $\log 2$ ratio and $-\log 10$ adjusted $P$-values are plotted and represented as volcano plots for $\mathrm{iCD}$ versus control, iUC versus control and $\mathrm{iCD}$ versus iUC comparisons in Figure 1D. For the non-inflamed tissue comparisons (iCD versus niCD and $\mathrm{iUC}$ versus niUC), the volcano plots are shown in Figure S4A,B in Additional file 2, respectively.
Differential gene expression analysis identified the following up/down-regulated genes: 761 and 278 proteincoding genes and 254 and 184 lncRNAs in iCD versus control and 1,085 and 599 protein-coding genes and 370 and 375 lncRNAs in iUC versus control (Table 3 and Figure 1E). The top up-regulated and down-regulated lncRNAs and protein-coding genes (based on FC) for $\mathrm{iCD}$ versus control and $\mathrm{iUC}$ versus control are listed in Tables 4 and 5. Interestingly, lncRNA RP11-731 F5.2 (whose 3' end partly spans the start of the IGHG2 gene) and antisense lncRNA $M M P 12$ were found significantly up-regulated, whereas the antisense DPP10-AS1, ANRIL (CDKN2B-AS1) and DIO3OS lncRNAs were significantly down-regulated in both $\mathrm{iCD}$ versus control and iUC versus control comparisons (Tables 4 and 5).

The top differentially expressed protein-coding genes included DUOXA2, CHI3L1, CXCL1 and SAA1, which were all significantly up-regulated, whereas, PCK1,SLC26A2, and GUCA2B were significantly down-regulated (Tables 4 and 5). In case of iCD versus controls, REG3A was $>52$ fold up-regulated (adjusted $P$-value $=2.17 \mathrm{e}-04$ ). The top differentially expressed lncRNAs and protein-coding genes for $\mathrm{iCD}$ versus niCD and $\mathrm{iUC}$ versus niUC comparisons displayed similar expression patterns as healthy controls (Tables S1 and S2 in Additional file 1).

On comparing niCD versus control and niUC versus control, only a small number of up/down-regulated genes (61 and 25 and 8 and 17 protein-coding, 12 and 19 and 9 and 10 lncRNA) were identified for niCD and niUC, respectively. Nearly all of the differentially expressed genes in niCD versus control were also present in the $\mathrm{iCD}$ versus control comparison with the exception of the proteincoding gene CRYBB2 (FC = -1.5; Table S3 in Additional file 1). Conversely, for niUC versus control, most (15 out of 17) of the up-regulated genes, including four small nucleolar RNAs (snoRNAs: SNORD97, SNORA28, SNORA53, and $S N O R A 74 A)$ and the down-regulated genes (MAST3, CPT1B, LOC338799, EXOC3L4, and MAPK8IP3) were specifically found in niUC only (Table S4 in Additional file 1). Importantly, in the iCD versus iUC comparison, 18 and 32 protein-coding genes and 13 and 10 lncRNAs were significantly found to be up/down-regulated, respectively. The top up/down-regulated lncRNAs and protein-coding genes for iCD versus iUC are shown in Table 6. Annotations for the Gencode v.15 [38] microarray features for lncRNAs are summarized in Figure 2A. Most of the differentially expressed lncRNAs identified in our analysis belonged to three main classes: antisense, processed transcripts and intergenic lincRNAs (Figure 2B), as described in the following section.

Additionally, we also tested the differences between the clinical subgroups for the top differentially expressed protein-coding and IncRNA genes. The top three upregulated protein-coding genes (DUOXA2, CHI3L1 and 


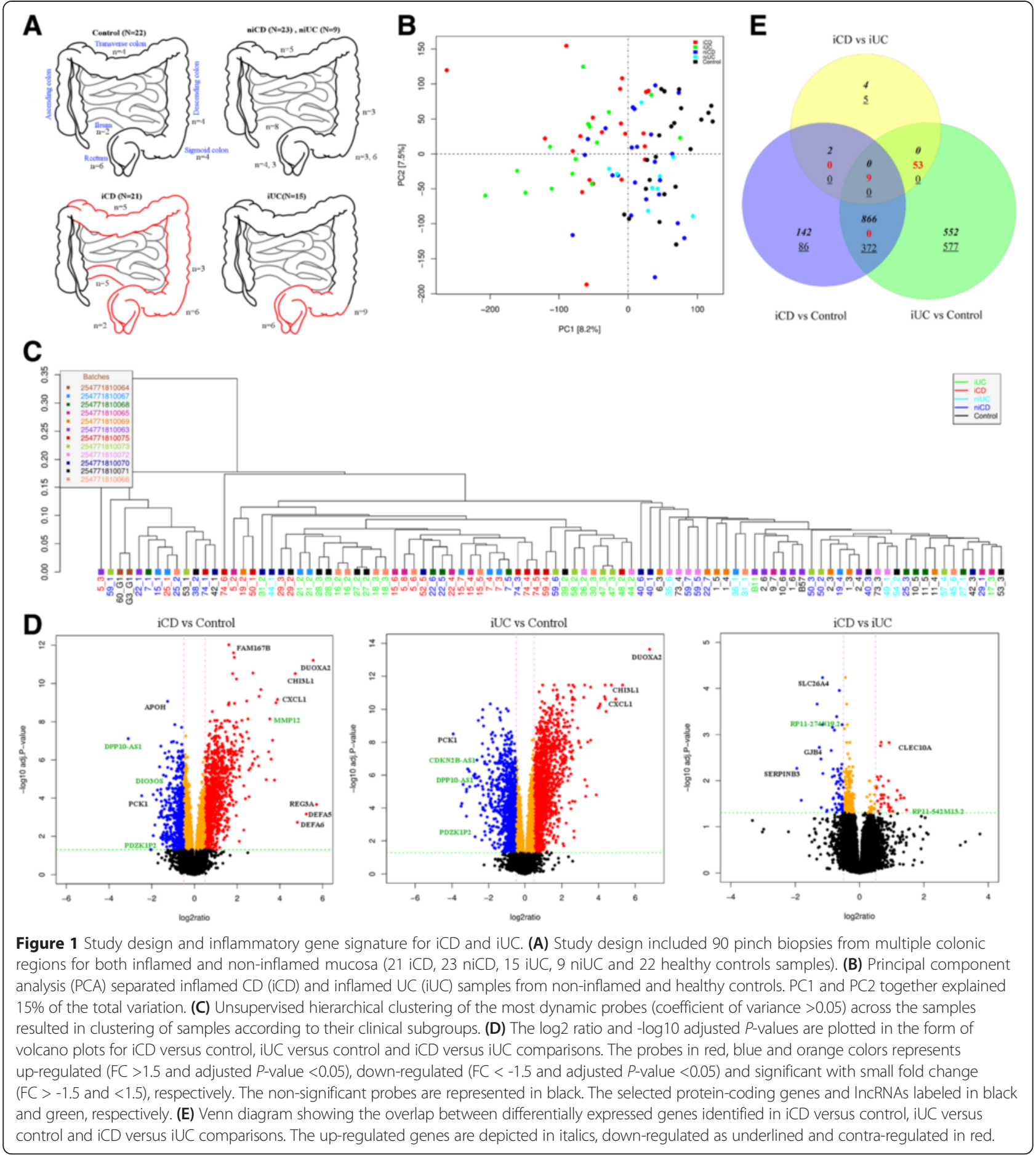

CXCL9) and IncRNA genes (MMP12, FAM66D and $S A A 2-S A A 4)$ showed increasing signal intensity based on the averaged gene expression levels across the spectrum of clinical subgroups from control to $\mathrm{iCD}$ and iUC $(P$-value $<0.001$; Figure $2 \mathrm{C})$. For the top three downregulated protein-coding genes (PCK1, GUCA2B and TNNC2) and lncRNA genes (DPP10-AS1, PDZK1P2 and $A N R I L)$, we observed decreasing signal intensity across the clinical subgroups from $\mathrm{iCD}$ and iUC to controls ( $P$-value $<0.001$; Figure $2 C)$. Importantly, eight major isoforms (out of a total of 17 annotated isoforms) of ANRIL were found to be down-regulated in iCD and iUC compared with controls and non-inflamed tissues in our data (Tables 4 and 5). ANRIL was -2.97-fold and -2.72-fold down-regulated in $\mathrm{iCD}$ versus control and $\mathrm{iCD}$ versus niCD comparisons, and -8.31-fold and -7.98-fold down- 
Table 2 Clinical parameters

\begin{tabular}{|c|c|c|c|}
\hline \multirow[t]{2}{*}{ Number of individuals } & $C D$ & UC & Controls \\
\hline & 13 & 20 & 12 \\
\hline $\begin{array}{l}\text { Age (median years } \\
\text { (range)) }\end{array}$ & 31 (19-59) & $46(18-68)$ & $54(18-77)$ \\
\hline $\begin{array}{l}\text { Average age at } \\
\text { diagnosis (years) }\end{array}$ & 27 & 33 & NA \\
\hline $\begin{array}{l}\text { Average years with } \\
\text { disease (disease } \\
\text { duration) }\end{array}$ & 8 & 9.3 & NA \\
\hline Female/male & $6 / 7$ & $13 / 7$ & $8 / 4$ \\
\hline \multicolumn{4}{|l|}{ Smoking } \\
\hline Smoker (S) & 8 & 1 & 4 \\
\hline Previous (P) & 4 & 8 & 1 \\
\hline Never (N) & 1 & 11 & 5 \\
\hline Not disclosed (ND) & - & - & 2 \\
\hline \multicolumn{4}{|l|}{ Ethnicity } \\
\hline Danish (DK) & 9 & 19 & 12 \\
\hline European (EU) & 1 & - & - \\
\hline Middle Eastern (ME) & 3 & 1 & - \\
\hline $\begin{array}{l}\text { Number of individuals } \\
\text { with family history of } \\
\text { other autoimmune } \\
\text { diseases }\end{array}$ & $1(7 \%)$ & $6(30 \%)$ & $5(41 \%)$ \\
\hline $\begin{array}{l}\text { Number of patients } \\
\text { on medication }\end{array}$ & & & NA \\
\hline 5-ASA & 2 & 13 & \\
\hline Solumedrol & 2 & 1 & \\
\hline Azathioprin & 2 & 2 & \\
\hline Budesonide & 1 & 1 & \\
\hline Prednisolon & 1 & 2 & \\
\hline Disease index & $H B$ index $=3-36$ & SCCAl index $=2-12$ & \\
\hline
\end{tabular}

Each column summarizes characteristics for all patients contributing samples to the corresponding sample groups. 5-ASA, 5-aminosalicylic acid; HB index, Harvey Bradshaw index; NA, not applicable; SCCAI index; Simple Clinical Colitis Activity index.

regulated in iUC versus control and iUC versus niUC comparisons, respectively.

Furthermore, for the validation of microarray results by qPCR, we selected eight top differentially expressed genes (based on FC) common between $\mathrm{iCD}$ versus control and iUC versus control (up-regulated: DUOXA2, CHI3L1, DUOX2, MMP12, RP11-731 F5.2; down-regulated: PCK1, DPP10-AS1, ANRIL). The qPCR analysis confirmed the microarray expression results with respect to the fold change values (Table S5 in Additional file 1). We also performed qPCR analysis for DUOX2, although it was not probed on our microarray, but it has been implicated along with its maturation factor, DUOXA2, in IBD pathogenesis (see Discussion). Both DUOXA2 and DUOX2 were found to be significantly up-regulated in $\mathrm{iCD}$ versus control ( $\mathrm{FC}=8.83$ and 5.85 , respectively) and iUC versus
Table 3 Total number of differentially expressed genes

\begin{tabular}{|c|c|c|c|c|}
\hline & iCD & niCD & iUC & niUc \\
\hline \multicolumn{5}{|c|}{ Total differentially expressed genes } \\
\hline Control & 1,477 & 73 & 2,429 & 44 \\
\hline iCD & & 435 & 73 & \\
\hline iUC & & & & 1,814 \\
\hline \multicolumn{5}{|c|}{ Protein-coding genes } \\
\hline Control & 1,039 & 61 & 1684 & 25 \\
\hline iCD & & 328 & 50 & \\
\hline iUC & & & & 1,215 \\
\hline \multicolumn{5}{|l|}{ IncRNAs } \\
\hline Control & 438 & 12 & 745 & 19 \\
\hline iCD & & 107 & 23 & \\
\hline iUC & & & & 599 \\
\hline
\end{tabular}

Total differentially expressed genes identified in seven pairwise comparisons (iCD versus control, iUC versus control, iCD versus niCD, iUC versus niUC, niCD versus control, niUC versus control and $\mathrm{iCD}$ versus iUC).

control (FC $=9.14$ and 6.05 , respectively) (Figure $2 \mathrm{D})$. For the remaining four comparisons, we also tested five differentially expressed genes by qPCR validation (Table S6 and S7 in Additional file 1).

\section{Overlap of differentially expressed genes in inflamed Crohn's disease and inflamed ulcerative colitis}

A Venn diagram illustrating the relationship between lncRNAs and protein-coding genes differentially expressed in iCD and iUC is shown in Figure 3. In total, 337 differentially expressed lncRNAs were identified as common between iCD and iUC with 100 unique lncRNAs for iCD and 400 unique lncRNAs for iUC (compared with the healthy controls; Figure 3A). For the protein-coding genes, 901 differentially expressed genes were found to be common between $\mathrm{iCD}$ and $\mathrm{iUC}$ with 128 unique for $\mathrm{iCD}$ and 739 unique for iUC (Figure 3B). Conversely, in the iCD versus iUC comparison, 19 out of 23 and 45 out of 50 differentially expressed lncRNAs and protein-coding genes, respectively, overlapped with $\mathrm{iCD}$ versus control and iUC versus control.

The unsupervised hierarchical clustering showed that both inflamed groups (iCD and $\mathrm{iUC}$ ) cluster together, in contrast to the non-inflamed groups (niCD and niUC), which clustered with healthy controls. The normalized gene expression values from the above-mentioned 337 IncRNAs and 901 protein-coding genes common to both $\mathrm{iCD}$ and iUC conditions were averaged for each of the five clinical subgroups and are visualized in a heat map in Figure 3C,D. The expression patterns for the specific up-regulated and down-regulated genes showed increasing or decreasing signal intensity across the clinical subgroups (from iCD, iUC, niCD, niUC and healthy controls). Collectively, these overlapping differentially expressed 
Table 4 Top 10 differentially expressed IncRNA and protein-coding genes in inflamed Crohn's disease

\begin{tabular}{|c|c|c|}
\hline Gene name & Transcript & FC \\
\hline \multicolumn{3}{|l|}{ Up-regulated IncRNAs } \\
\hline RP11-731 F5.2 & ENST00000460164.1 & 14.14 \\
\hline MMP12 & ENST00000532855.1 & 6.64 \\
\hline MMP12 & ENST00000326227.5 & 6.52 \\
\hline RP11-465 L10.10 & ENST00000419897.1 & 5.69 \\
\hline RP11-44 K6.2 & ENST00000520185.1 & 3.83 \\
\hline FAM66D & ENST00000526690.1 & 3.36 \\
\hline LINC01272 & ENST00000445003.1 & 3.35 \\
\hline RP11-44 K6.4 & ENST00000522970.1 & 3.24 \\
\hline SAA2-SAA4 & ENST00000524555.1 & 3.16 \\
\hline KIF9-AS1 & ENST00000429315.2 & 3.14 \\
\hline \multicolumn{3}{|l|}{ Down-regulated IncRNAs } \\
\hline DPP10-AS1 & ENST00000432658.1 & -8.57 \\
\hline PDZK1P2 & ENST00000401008.2 & -4.11 \\
\hline DIO3OS & ENST00000553575.1 & -3.01 \\
\hline DIO3OS & ENST00000554694.1 & -3.01 \\
\hline DIO3OS & ENST00000557532.1 & -2.99 \\
\hline DIO3OS & ENST00000557109.1 & -2.98 \\
\hline ANRIL (CDKN2B-AS1) & ENST00000422420.1 & -2.97 \\
\hline ANRIL (CDKN2B-AS1) & ENST00000428597.1 & -2.97 \\
\hline DIO3OS & ENST00000554441.1 & -2.96 \\
\hline DIO3OS & ENST00000554735.1 & -2.95 \\
\hline \multicolumn{3}{|c|}{ Up-regulated protein-coding genes } \\
\hline REG3A & NM_138938 & 52.71 \\
\hline DUOXA2 & NM_207581 & 47.26 \\
\hline DEFA5 & NM_021010 & 37.73 \\
\hline DEFA6 & NM_001926 & 28.33 \\
\hline CHI3L1 & NM_001276 & 26.29 \\
\hline CXCL1 & NM_001511 & 14.8 \\
\hline DMBT1 & NM_007329 & 13.45 \\
\hline SAA1 & NM_000331 & 12.67 \\
\hline CXCL9 & NM_002416 & 12.07 \\
\hline IGHG3 & ENST00000390551 & 11.52 \\
\hline \multicolumn{3}{|c|}{ Down-regulated protein-coding genes } \\
\hline PCK1 & NM_002591 & -5.55 \\
\hline SLC26A2 & NM_000112 & -3.82 \\
\hline C10orf116 & NM_006829 & -3.8 \\
\hline GUCA2B & NM_007102 & -3.61 \\
\hline LCN15 & NM_203347 & -3.43 \\
\hline
\end{tabular}

Table 4 Top 10 differentially expressed IncRNA and protein-coding genes in inflamed Crohn's disease (Continued)

\begin{tabular}{lll}
\hline AQP7P1 & NR_002817 & -3.32 \\
TRPM6 & NM_017662 & -3.19 \\
TNNC2 & NM_003279 & -3.1 \\
UGT2A3 & NM_024743 & -2.97 \\
ADH1C & NM_000669 & -2.96 \\
\hline
\end{tabular}

Top 10 up- and down-regulated IncRNAs and protein-coding genes in iCD versus control comparison. The log2 fold change is denoted as FC.

genes between $\mathrm{iCD}$ versus control and iUC versus control define a distinct inflammatory $\mathrm{iCD} / \mathrm{iUC}$ gene expression signature. Importantly, this inflammatory gene signature included the key drivers of the innate and adaptive immune responses (for example, DUOXA2 and CXCL1).

\section{Comparison of expression levels of top differentially} expressed genes in patients and healthy controls

To stratify iCD and iUC samples from the healthy controls, we also compared the expression profiles of the top 20 up/down-regulated lncRNAs and the top 20 up/downregulated protein-coding genes (based on FC) through unsupervised hierarchical clustering. An expression map of these top 40 differentially expressed genes displayed a clear separation of the patients from the control groups (Figure 4A,B), except for the two iUC samples B11 and 17_3, which were misclassified in the clustering. The magnitude of $\log 2$ intensity signal for these top differentially expressed genes displayed in Figure 4 was $>6$ in both iCD and iUC. Interestingly, in the case of the iCD versus iUC comparison, clustering was unable to distinguish between iCD and iUC patients (Figure S5 in Additional file 2). In addition to the top candidates, we also compared the expression profiles of all differentially expressed lncRNAs and protein-coding genes, and observed similar results as described above (Figure S6A,B in Additional file 2).

\section{Inflammatory response and antimicrobial peptide genes} are dysregulated in inflamed Crohn's disease and inflamed ulcerative colitis

Antimicrobial peptides (AMPs) play an important role in protecting the host intestinal mucosa against microorganisms and AMP dysregulation has been associated with IBD pathogenesis (see Discussion for details). Therefore, we investigated whether there were differences in the expression of genes involved in the inflammatory response and AMP production between the different clinical subgroups. Our analysis identified key genes associated with the inflammatory response, including the pro-inflammatory chemokines and cytokines. CCL11, CCL19, CCL4 and CXCL9 were significantly up-regulated in both $\mathrm{iCD}$ versus control and iUC versus control. In addition, we also found 
Table 5 Top 10 differentially expressed IncRNA and protein-coding genes in inflamed ulcerative colitis

\begin{tabular}{l} 
Gene name \\
\hline Up-regulated IncRNAs \\
RP11-731 F5.2 \\
MMP12 \\
MMP12 \\
RP11-465 L10.10 \\
KIF9-AS1 \\
FAM66D \\
SAA2-SAA4 \\
CLRN1-AS1 \\
RP11-1149O23.3 \\
RP5-1028 K7.2 \\
Down-regulated IncRNAs \\
ANRIL (CDKN2B-AS1) \\
ANRIL (CDKN2B-AS1) \\
ANRIL (CDKN2B-AS1) \\
ANRIL (CDKN2B-AS1) \\
ANRIL (CDKN2B-AS1) \\
ANRIL (CDKN2B-AS1) \\
ANRIL (CDKN2B-AS1) \\
PDZK1P2 \\
DPP10-AS1 \\
ANRIL (CDKN2B-AS1)
\end{tabular}

Up-regulated protein-coding genes

DUOXA2

CHI3L1

SAA1

CXCL1

MMP7

SLC6A14

IGHG3

MMP12

C4orf7

CXCL2

Down-regulated protein-coding genes

PCK1

OSTalpha

ANPEP

SLC26A2

GBA3

Transcript FC

ENST00000460164.1 20.64

ENST00000532855.1 17.05

ENST00000326227.5 16.54

ENST00000419897.1 9.52

ENST00000429315.2 5.75

ENST00000526690.1 5.73

ENST00000524555.1 5.66

ENST00000476886.1 4.64

ENST00000517774.1 4.29

ENST00000578280.1 4.21

ENST00000422420.1 - -8.67

ENST00000428597.1 -8.31

ENST00000585267.1 $\quad-7.06$

ENST00000580576.1 -6.92

ENST00000577551.1 -6.74

ENST00000581051.1 - -6.72

ENST00000582072.1 -6.68

ENST00000401008.2 - -6.67

ENST00000432658.1 $\quad-5.95$

ENST00000421632.1 -5.78

NM_207581 $\quad 109.61$

NM_001276 $\quad 39.71$

NM_000331 $\quad 30.67$

NM_001511 25.92

NM_002423 21.2

NM_007231 20.52

ENST00000390551 20.14

NM_002426 $\quad 15.76$

NM_152997 $\quad 14.76$

NM_002089 $\quad 11.91$

NM_002591 - -15.24

NM_152672 -11.33

NM_001150 - -11.02

NM_000112 - -10.46

NM_020973 $\quad-9.28$
Table 5 Top 10 differentially expressed IncRNA and protein-coding genes in inflamed ulcerative colitis (Continued)

\begin{tabular}{llr}
\hline GUCA2A & NM_033553 & -9.22 \\
SLC3A1 & NM_000341 & -9.21 \\
GUCA2B & NM_007102 & -8.84 \\
TMIGD1 & NM_206832 & -8.17 \\
SLC1A7 & NM_006671 & -6.57 \\
\hline Top 10 up- and down-regulated IncRNA and protein-coding genes in iUC \\
versus control comparison. The log2 fold change is denoted as FC.
\end{tabular}

key antimicrobial response genes to be significantly upregulated in $\mathrm{iCD}$ and iUC compared with healthy controls (Figure 5). REG3A, DEFA5 and DEFA6 were $>30$-fold upregulated only in $\mathrm{iCD}$ versus control. The chemokines CXCL1 and CXCL2 were $>15$ - and $>25$-fold up-regulated in both $\mathrm{iCD}$ versus control and iUC versus control, respectively. CXCL5, IL15 and C3AR1 were specifically upregulated in $\mathrm{ICD}$ versus control (Figure 5). Notably, the NOD2 gene was $>2$-fold up-regulated in iCD versus control, iUC versus control and $\mathrm{iCD}$ versus niCD comparisons. DEFB1 and NPY were the only AMP genes that were significantly down-regulated in both iUC and $\mathrm{iCD}$.

Differentially expressed genes in inflamed Crohn's disease and inflamed ulcerative colitis are enriched within inflammatory bowel disease loci

Since most disease-associated susceptibility SNPs map to the non-coding regions in the genome, we looked for the presence of known IBD-associated SNPs (total of 233 SNPs) within the Gencode v.15 annotated lncRNAs. Interestingly, 29 IBD risk variants intersected 37 lncRNAs, of which only the IFNG-AS1 antisense lincRNA (ENST00000536914.1) harboring the UC susceptibility SNP rs7134599 was found to be differentially expressed in our study. IFNG-ASI was up-regulated in iUC versus control $(\mathrm{FC}=1.54)$ and iUC versus niUC $(\mathrm{FC}=1.52)$. Furthermore, we identified IBD loci-associated lncRNAs and protein-coding genes by intersecting the IBD susceptibility loci, which was defined as a $500 \mathrm{~kb}$ long genomic region with the IBD risk variant in the middle. In total, 1,040 IBD loci-associated lncRNAs were identified, out of which 96 lncRNAs were found to be differentially expressed (Table S8 in Additional file 1). These differentially expressed lncRNAs co-localized with 57 IBD risk variants (within a $500 \mathrm{~kb}$ locus), and were found to be enriched within IBD loci $(P$-value $<0.0001$, Pearson's Chi-squared test). In the case of protein-coding genes, 681 genes were found to be associated with IBD loci, out of which 154 were differentially expressed and enriched within IBD loci ( $P$-value $<0.0001$, Pearson's Chisquared test). Unsupervised hierarchical clustering of averaged and normalized gene expression values of 96 and 154 differentially expressed IBD loci-enriched lncRNAs and 
Table 6 Top 10 differentially expressed genes in inflamed Crohn's disease versus inflamed ulcerative colitis

\begin{tabular}{|c|c|c|}
\hline Gene name & Transcript & FC \\
\hline \multicolumn{3}{|c|}{ Up-regulated IncRNAs } \\
\hline FLJ42969 & ENST00000514926.1 & 2.6 \\
\hline AC007182.6 & ENST00000455232.1 & 2.42 \\
\hline RP11-542 M13.2 & ENST00000599411.1 & 2.04 \\
\hline RP11-399 F4.4 & ENST00000453998.1 & 1.87 \\
\hline FAM95B1 & ENST00000455995.1 & 1.87 \\
\hline RP3-395 M20.8 & ENST00000432521.2 & 1.69 \\
\hline RP3-395 M20.8 & ENST00000448624.2 & 1.65 \\
\hline OPLAH & ENST00000426825.1 & 1.61 \\
\hline OPLAH & ENST00000534424.1 & 1.61 \\
\hline SPPL2B & ENST00000592738.1 & 1.59 \\
\hline \multicolumn{3}{|c|}{ Down-regulated IncRNAs } \\
\hline AL928742.12 & ENST00000412518.1 & -2.01 \\
\hline RP11-444D3.1 & ENST00000540811.1 & -1.84 \\
\hline AL928742.12 & ENST00000427543.1 & -1.8 \\
\hline FAM25D & ENST00000426412.2 & -1.69 \\
\hline RP11-274 N19.2 & ENST00000515643.1 & -1.64 \\
\hline RP11-838 N2.4 & ENST00000579007.1 & -1.59 \\
\hline RP11-279 F6.3 & ENST00000558941.1 & -1.57 \\
\hline RP11-279 F6.3 & ENST00000559212.1 & -1.55 \\
\hline LINC00524 & ENST00000555860.1 & -1.54 \\
\hline VAV3-AS1 & ENST00000438318.1 & -1.52 \\
\hline \multicolumn{3}{|c|}{ Up-regulated protein-coding genes } \\
\hline C8G & NM_000606 & 2.75 \\
\hline SLC25A34 & NM_207348 & 2.43 \\
\hline UGT1A6 & NM_001072 & 2.25 \\
\hline LRRC66 & NM_001024611 & 2.20 \\
\hline EXOC3L4 & NM_001077594 & 1.95 \\
\hline ANO7 & NM_001001891 & 1.95 \\
\hline GLYCTK & NM_145262 & 1.90 \\
\hline CLEC10A & NM_182906 & 1.89 \\
\hline FAM95B1 & NR_026759 & 1.89 \\
\hline LPIN3 & NM_022896 & 1.87 \\
\hline \multicolumn{3}{|c|}{ Down-regulated protein-coding genes } \\
\hline SERPINB3 & NM_006919 & -3.87 \\
\hline SLC6A14 & NM_007231 & -3.51 \\
\hline GAL & NM_015973 & -2.50 \\
\hline GJB4 & NM_153212 & -2.38 \\
\hline IGHV1-58 & ENST00000390628 & -2.29 \\
\hline
\end{tabular}

Table 6 Top 10 differentially expressed genes in inflamed Crohn's disease versus inflamed ulcerative colitis (Continued)

\begin{tabular}{lll}
\hline CRYM & NM_001888 & -2.28 \\
SLC26A4 & NM_000441 & -2.22 \\
DEFB103B & NM_018661 & -2.22 \\
LAMC2 & NM_005562 & -2.20 \\
TUSC3 & NM_178234 & -2.02 \\
\hline
\end{tabular}

Top 10 up- and down-regulated IncRNAs and protein-coding genes in iCD versus iUC. The log2 fold change is denoted as FC.

protein-coding genes, respectively, enabled independent stratification of disease from the controls and further distinguished inflamed from non-inflamed conditions in both CD and UC (Figure 6).

\section{Regulatory IBD-associated SNPs co-localize with} differentially expressed IBD loci-associated IncRNAs Next, we asked whether active regulatory regions within the IBD loci overlap with the differentially expressed IBD loci-associated lncRNAs. IBD-associated SNPs overlapping active regulatory elements in intestinal epithelium were retrieved from the Mokry et al. study [7]. In their study, the active regions overlapping IBD-associated SNPs were identified based on H3K27ac chromatin immunoprecipitation and sequencing (ChIP-Seq). Out of 96 differentially expressed IBD loci-associated lncRNAs, 68 were found to be associated with 24 IBD loci SNPs colocalizing with the active regulatory elements in intestinal epithelium and immune cells (Table S8 in Additional file 1 ). These overlapping IBD loci-associated active regulatory elements have been reported to frequently co-localize with the known transcription factor binding motifs [7]. A number of IBD-associated SNPs potentially affect the binding affinity of transcriptional factors, and thus perturb the gene expression. Additionally, IBD-associated risk variants also act as expression quantitative trait loci (eQTLs) signals for a number of genes (Table S8 in Additional file 1). For example, IBD-associated risk variant rs10797432, located within the IBD loci-associated lncRNA RP3-395 M20.8 (ENSG00000238164), alters the binding motifs for TFAP2A and CTCF. Furthermore, it is also known to act as a cis-eQTL for MMEL1 in monocytes. The regulatory IBD-associated SNP rs1569723, located within the IBD lociassociated lncRNA RP11-465 L10.10 (ENSG00000204044), acts as a cis-eQTL for CD40 in monocytes. Also, SNP rs12946510, associated with lncRNAs RP11-387H17.4 (ENSG00000264968) and RP11-94 L15.2 (ENSG00000 264198), is known to perturb the binding sites for transcription factors FOXO1, ELF3, and SRF. In addition, this SNP also acts as a cis-eQTL for the pseudogene KRT222P, transcriptional co-activator complex component MED24, transcription factor NR1D1, and ORMDL3 in lympho- 

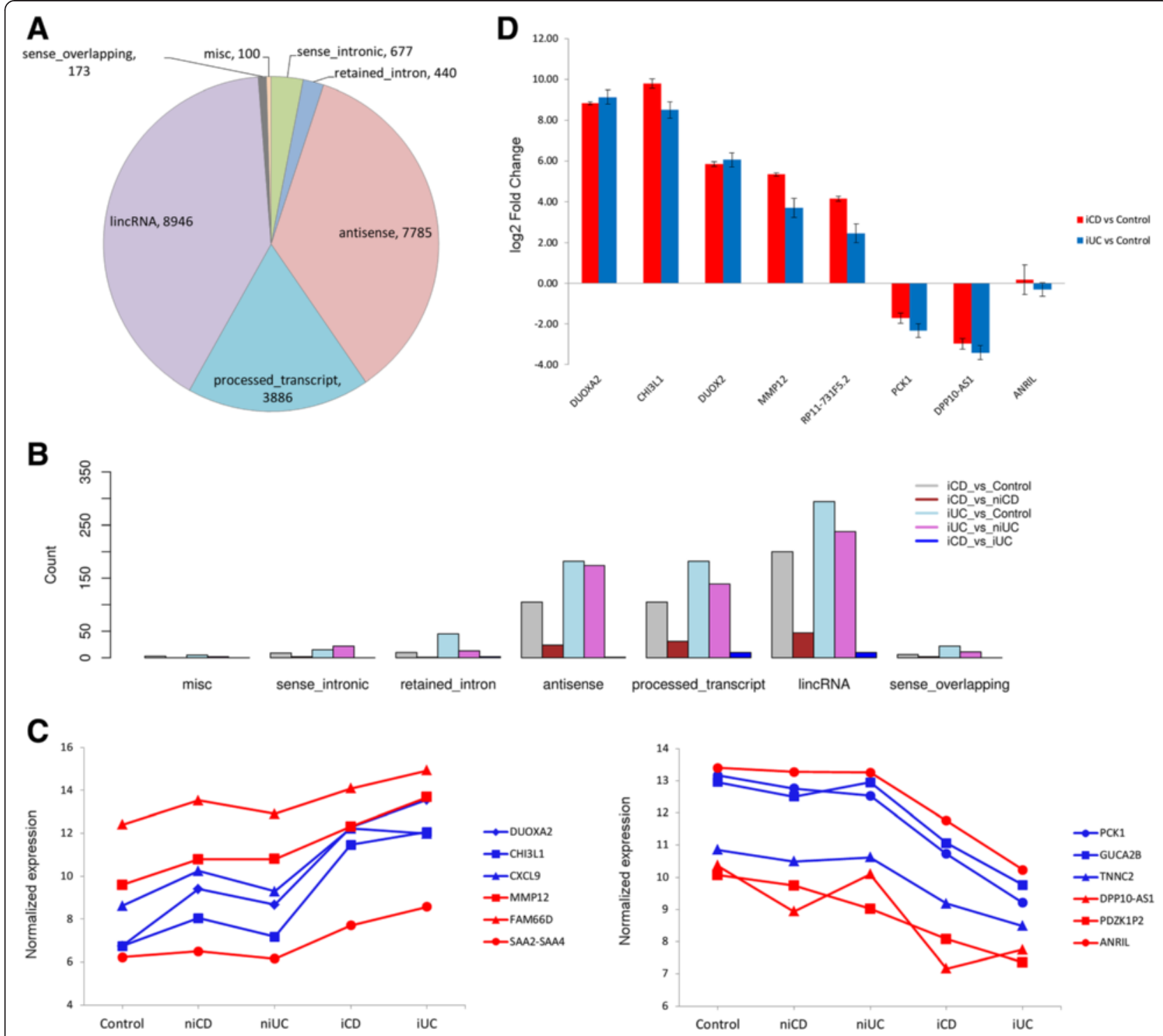

Figure 2 Gencode v.15 annotation of the total differentially expressed IncRNAs in IBD and microarray validation by qPCR. (A) The Gencode v.15 array targeted 22,007 IncRNA transcripts falling into seven major annotation classes (antisense, processed transcripts, intergenic (lincRNAs), sense overlapping, sense intronic and retained introns). Three classes (ambiguous_orf, non-coding RNAs and TEC (to be experimentally confirmed) with small number of IncRNAs were merged into a miscellaneous (misc) class for better representation. (B) Three major classes of differentially expressed IncRNAs identified in our study: intergenic (lincRNAs), processed transcripts and antisense IncRNAs. (C) Differences between the expression levels of the top three most up- and down-regulated protein-coding genes (in blue) and IncRNA genes (in red) were tested using Kruskal-Wallis test with Dunn's multiple comparison test. The top three up-regulated protein-coding genes (DUOXA2, CHI3L1 and CXCL9) and IncRNA genes (MMP12, FAM66D and SAA2-SAA4) showed increasing signal intensity from control group to inflamed CD and UC groups based on averaged gene expression levels $(P$-value $<0.001)$. The top three down-regulated protein-coding genes (PCK1, GUCA2B and TNNC2) and IncRNA genes (DPP10-AS1, PDZK1P2 and ANRIL) showed decreasing signal intensity across the clinical subgroups from iCD and iUC to controls ( $P$-value $<0.001)$. (D) A total of eight genes were selected for real-time PCR validation of the microarray data in ICD versus control (red) and iUC versus control (blue). The log2 fold change is plotted on the y axis.

blastoid cell lines. In the case of the antisense lncRNA CTD-2196E14.5 (ENSG00000261266), the associated SNP rs7404095 acts as a cis-eQTL for PRKCB in lymphoblastoid cell lines and PRKCB1 in monocytes. Moreover, SNP rs734999, associated with lncRNA RP3-395 M20.8 (ENSG00000238164), acts as a cis-eQTL for TNFRSF14 in lymphoblastoid cell lines.
Cis-acting correlation of expression between differentially expressed inflammatory bowel disease loci-associated IncRNAs and protein-coding genes

We computed pairwise Pearson correlations in order to explore the possible co-expression patterns between IBD loci-associated differentially expressed lncRNAs and protein-coding genes. Pairwise correlations of expression 


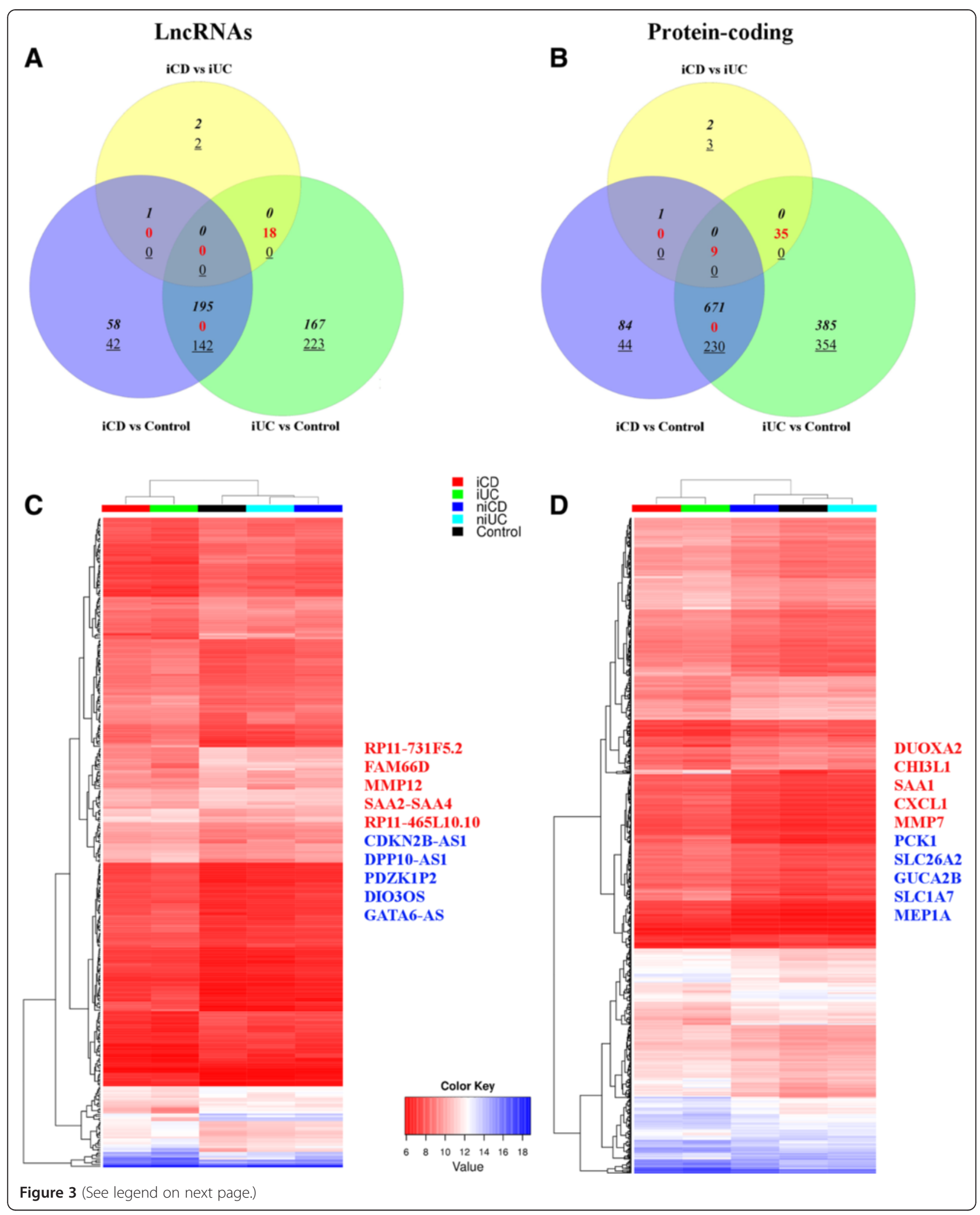


(See figure on previous page.)

Figure 3 Overlap of differentially expressed IncRNAs and protein-coding genes between iCD and iUC. (A,B) Venn diagrams show an overlap of 337 IncRNAs (A) and 901 protein-coding genes (B) that were differentially expressed (FC >1.5, adjusted $P$-value $<0.05$ ) between patients with iCD and iUC compared with healthy controls. We observed contra-regulated genes between iCD/iUC versus control comparisons compared with the iCD versus iUC comparison. The up-regulated genes are depicted in italics, down-regulated as underlined and contra-regulated in red. (C,D) Heat maps of average normalized gene expression for the overlapping 337 IncRNAs (C) and 901 protein-coding genes (D) between iCD and iUC in the five clinical subgroups (iCD, niCD, iUC, niUC and controls) are displayed. Selected up-regulated and down-regulated genes are listed.

involving neighboring lncRNAs and protein-coding genes associated with each IBD-associated SNP (500 kb loci with the SNP in the middle) were computed. We found positive $\left(r^{2} \geq 0.5\right)$ and extremely positive $\left(r^{2} \geq 0.9\right)$ correlations between the overlapping as well as cis-neighboring differentially expressed IBD loci-associated lncRNA-protein-coding gene pairs $(P$-value $<0.05)$. The pairwise correlations for six intersecting IBD loci-associated lncRNA-protein-coding gene pairs - LSP1 and ENST00000509204.1 (rs907611), HLA-DQB1 and ENST00000443574.1 (rs9268853, rs692 7022), MST1 and ENST00000563780.1 (rs9822268 and rs3197999), TSPAN33 and ENST00000498745.1 (rs472 8142), SLC22A5 and ENST00000417795.1 (rs2188962, rs12521868), DGRD and ENST00000442524.1 (rs1299 4997, rs3792109) - are plotted in Figure 7. Interestingly, lncRNA ENST00000563780.1 and MST1 protein-coding gene exhibited extremely positive correlation $\left(r^{2} \geq 0.99\right.$; Figure 7). Enrichment for positive correlations has been reported for the lncRNAs intersecting protein-coding genes in an antisense orientation [35]. Indeed, we also observed strong positive correlation $\left(r^{2} \geq 0.7\right)$ for the intersecting antisense lncRNA ENST00000417795 and the protein-coding gene SLC22A5.

\section{Functional annotation of differentially expressed IncRNAs}

The functional annotations of lncRNAs have mostly been based on the nearest-neighbor approach, that is, 'guilt-by-association' analyses - for example, Cabili et al. [39]. We therefore analyzed the GO terms of genes that overlap with or are neighbors of the differentially expressed lncRNAs. We identified 516 nearest proteincoding neighbors within a span of $<10 \mathrm{~kb}$ covering 610 differentially expressed lncRNAs. In addition, we also included 712 neighboring protein-coding genes for the 57 IBD risk variants (associated with 96 differentially expressed IBD loci-associated lncRNAs) based on a 1 $\mathrm{Mb}$ locus size for each variant. The most significant over-represented GO terms in the biological process category included antigen processing and presentation $(P$-value $7.39 \mathrm{e}-08)$, immune system process $(P$-value

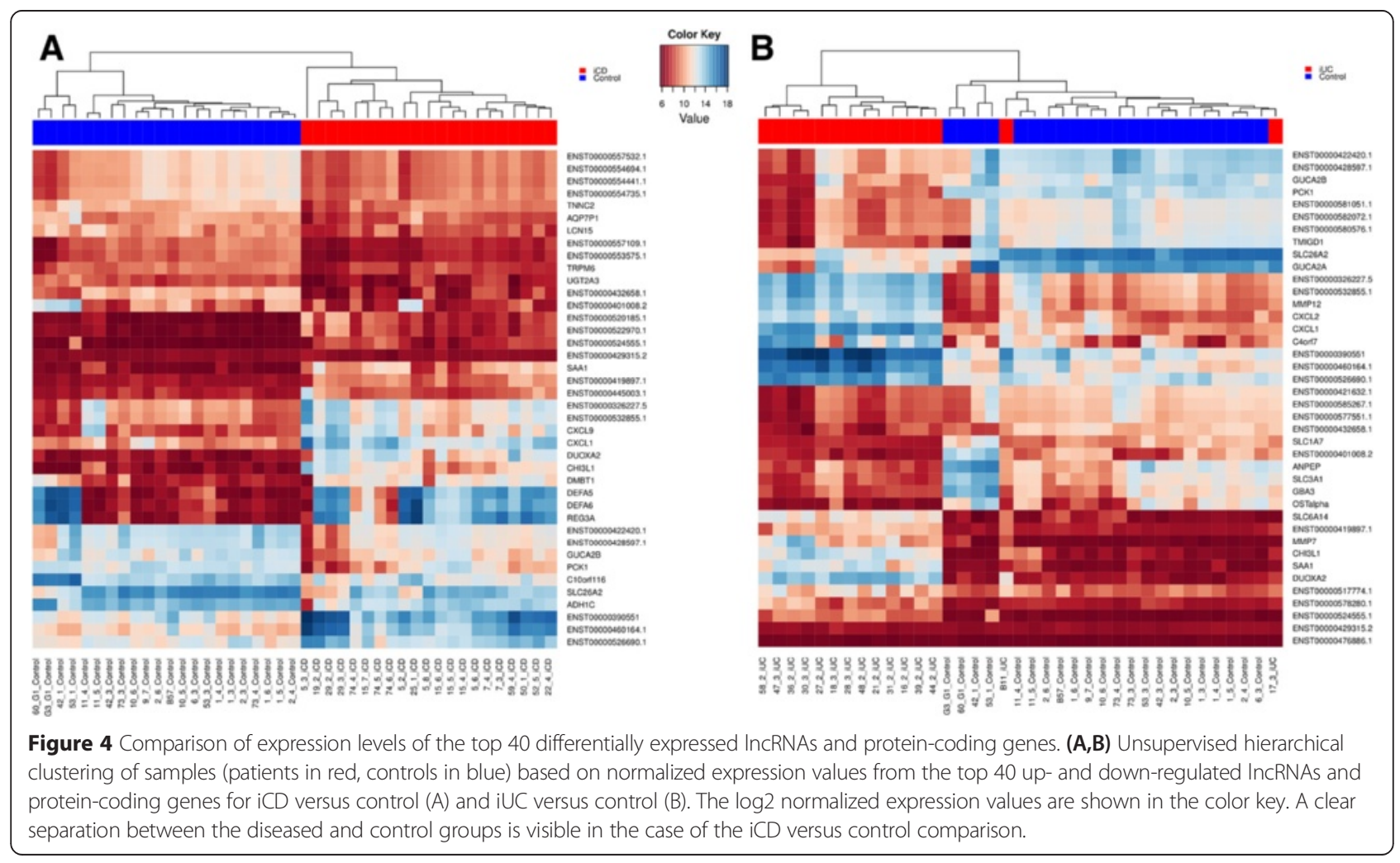




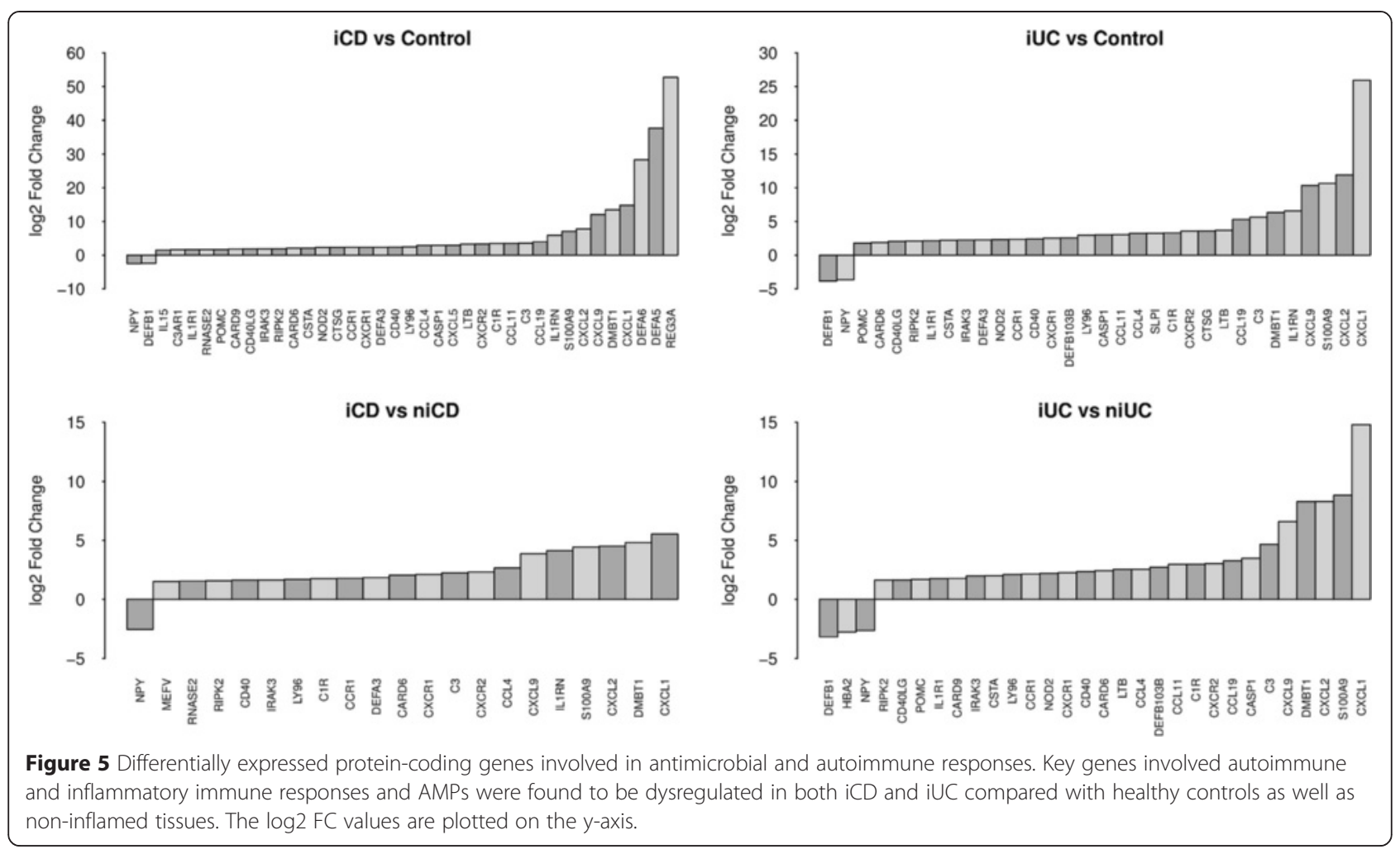

2.5e-05) and natural killer cell activation ( $P$-value 9.6e-05) (Table S9 in Additional file 1). In the cellular component category, we found enrichment for the MHC protein complex ( $P$-value 5.95e-09). Furthermore, we also observed enrichment for over-represented GO terms in the molecular function category, which included protein binding, receptor binding and cytokine activity.

Cross-validation of differentially expressed genes by SVM SVM [33] was used for classifying IBD cases from controls and for cross-validating differentially expressed genes identified by LIMMA. The best SVM classifier performance was obtained from differentially expressed lncRNAs identified in the iCD versus control followed by iUC versus control comparison (see Methods for details). The classifier distinguished $\mathrm{iCD}$ and iUC from controls with $100 \%$ and $94.6 \%$ accuracy, $100 \%$ and $100 \%$ specificity and $100 \%$ and $86.7 \%$ sensitivity, respectively. In addition, the classifier was also able to distinguish iCD and iUC from niCD and niUC samples with an accuracy of $86.4 \%$ and $91.7 \%$, specificity of $78.3 \%$ and $88.9 \%$ and sensitivity of $95.2 \%$ and $83.3 \%$, respectively. For the iCD versus iUC comparison, the accuracy of the classifier was $77.8 \%$, with $60.0 \%$ specificity and $90.4 \%$ sensitivity (Figure $8 \mathrm{~A}$ ). For the differentially expressed protein-coding genes, the classifier achieved an accuracy of $100 \%$ and $94.6 \%$, with $100 \%$ and $100 \%$ specificity, and $100 \%$ and $86.7 \%$ sensitivity, in discriminating $\mathrm{iCD}$ and $\mathrm{iUC}$ from controls, respectively
(Figure 8B). Similar to the above described observations, the classifier also allowed iCD and iUC to be distinguished from niCD and niUC samples with an accuracy of $81.8 \%$ and $83.3 \%$, specificity of $78.2 \%$ and $77.8 \%$ and sensitivity of $85.7 \%$ and $86.7 \%$, respectively. For the iCD versus iUC comparison, the accuracy of the classifier was $88.9 \%$, with $80.0 \%$ specificity and $95.2 \%$ sensitivity (Figure $8 \mathrm{~B}$ ). Furthermore, our classifier achieved a similar performance when using combined differentially expressed proteincoding and lncRNA genes or only protein-coding genes (data not shown). The effect of clinical parameters (Table 2; Figure S7 in Additional file 2) on disease (iCD or iUC) was described by the following linear function:

$$
\begin{aligned}
y= & 0.511+\left(-0.212^{*} \text { age }\right)+\left(-0.114^{*} \text { sex }\right) \\
& +\left(-0.339^{*} \text { smoking }\right)+\left(1.185^{*} \text { disease score }\right) \\
& +\left(-0.058^{*} \text { biopsy location }\right)
\end{aligned}
$$

Using t-statistics, $P$-values for linear regression coefficients for age, sex, smoking, disease index and biopsy location were $1.40 \mathrm{e}-01,2.29 \mathrm{e}-01,2.98 \mathrm{e}-03,5.02 \mathrm{e}-07$ and $6.43 \mathrm{e}-01$, respectively. Our analysis indicated that disease index had the strongest effect on defining $\mathrm{iCD}$ and $\mathrm{iUC}$ phenotype, followed by smoking, sex and age (Figures S7 and S8 in Additional file 2). Differentially expressed genes identified by LIMMA were verified by SVM-RFE [35], which revealed a robust concordance rate in terms 


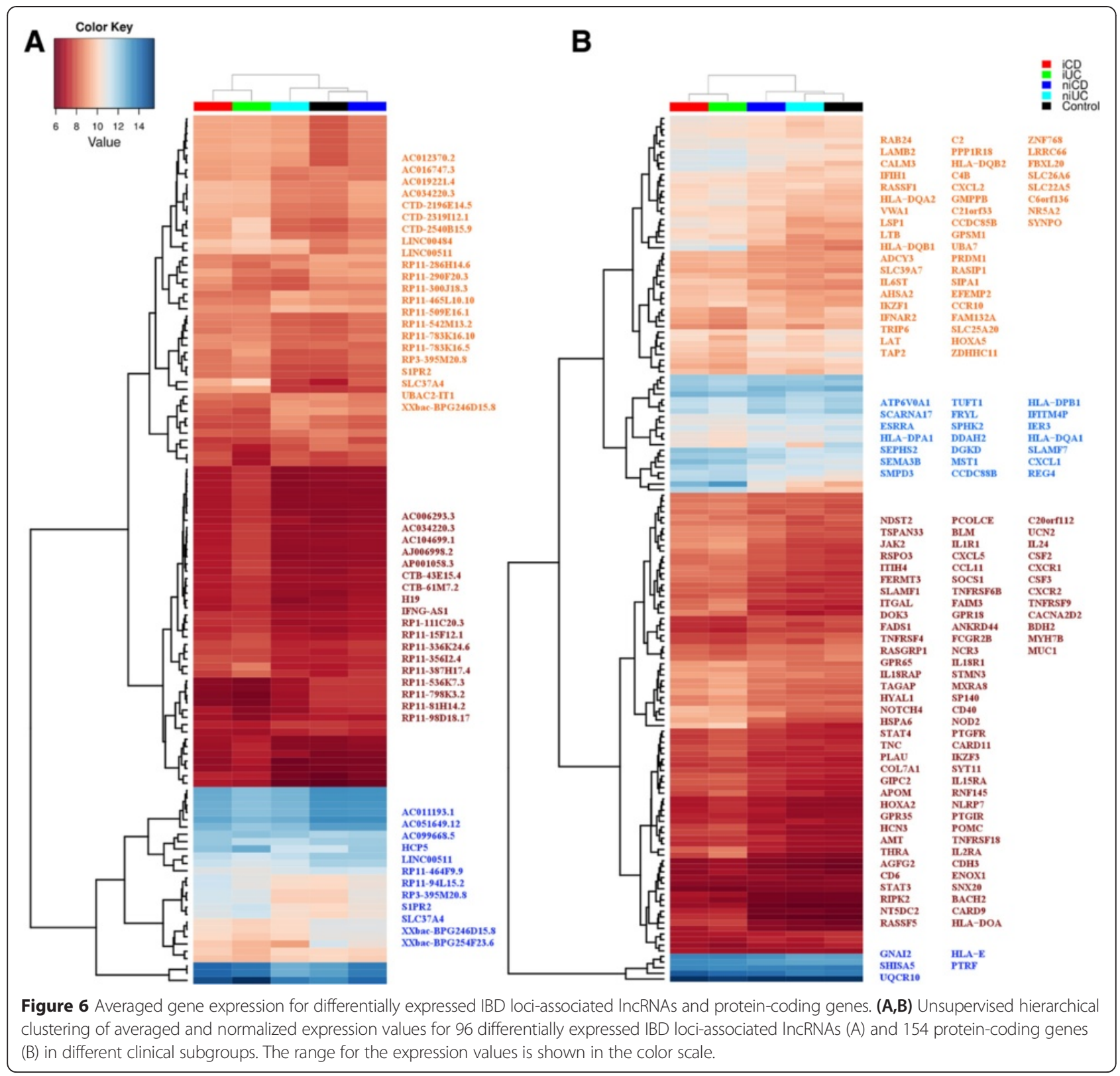

of total number of overlapping differentially expressed genes identified by the two methods (Figure S9 in Additional file 2). In both the $\mathrm{iCD}$ versus control and iUC versus control comparisons, an overlap of about $66 \%$ was observed. To control for any input bias, a randomized lncRNA gene list of the same size as the differentially expressed lncRNAs was also used in this analysis (Figure S8 in Additional file 2).

\section{Impact of clinical parameters on disease diagnosis}

We next investigated the impact of the clinical parameters for disease diagnosis, and whether expression profiles of differentially expressed genes are also correlated with other clinical parameters. The applied strategies were linear regression model and WGCNA [36]. The regression analysis showed a strong impact of the disease index (Harvey-Bradshaw Index for CD and Simple Clinical Colitis Activity Index for UC; $P$-value $<10 \mathrm{e}-6, t$-test), which by definition is positively correlated with the severity of the disease, and a significant impact of smoking ( $P$-value $<0.05, t$-test), which was, however, 3.5 times lower than the disease index and lower than the error rate. However, biopsy location did not show any significant effect on the severity of the disease. In agreement, the network analysis identified 10,435 genes significantly correlated with the disease index ( $P$-value $<0.05, t$-test), which is a clinical parameter with most related gene expression profiles. However, only 509 of these genes were differentially 

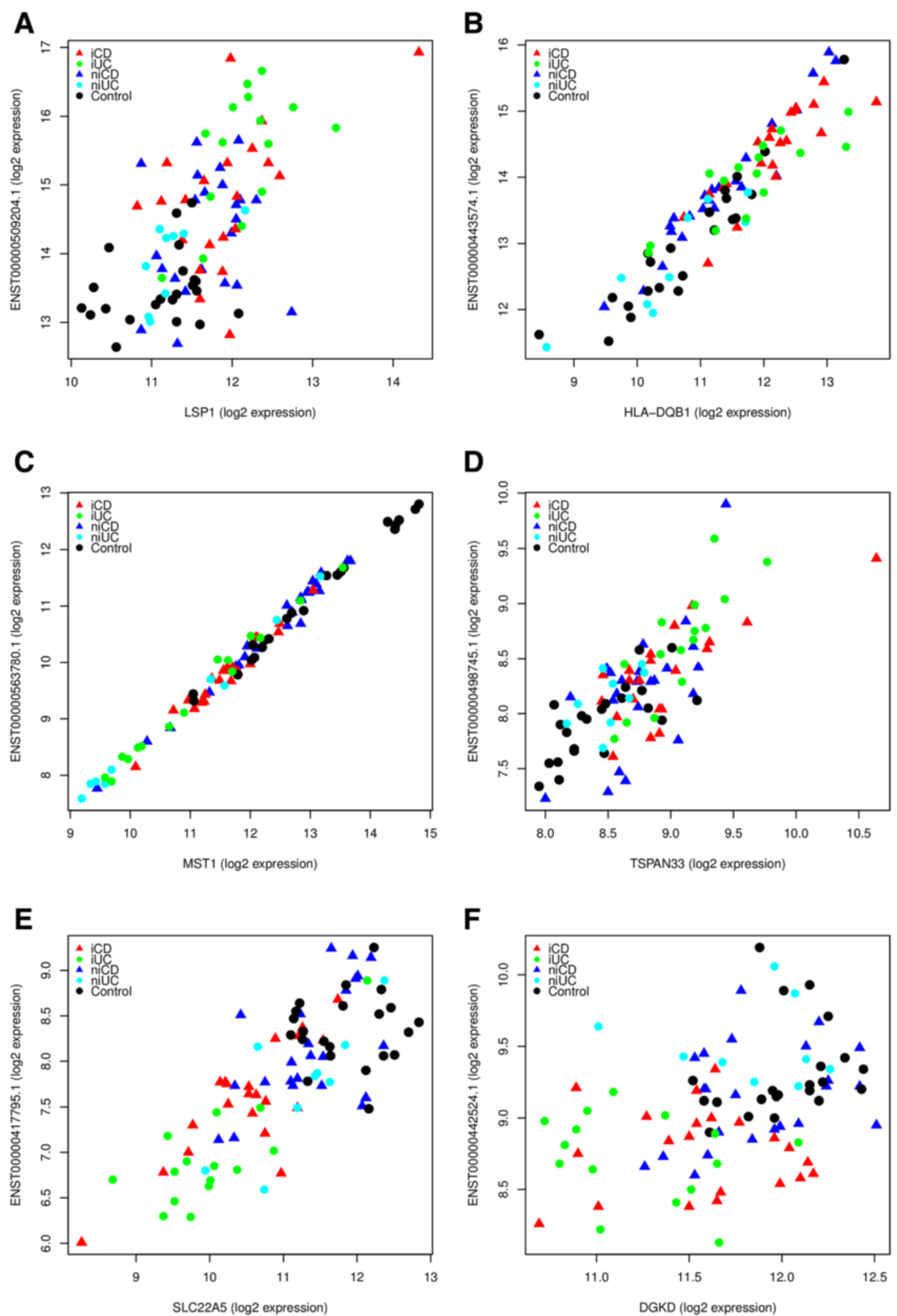

Figure 7 (See legend on next page.) 
(See figure on previous page.)

Figure 7 Correlations of expression for cis-neighboring pairs of IBD loci-associated differentially expressed IncRNAs and protein-coding genes. (A-F) Overall positive correlations between overlapping protein-coding and IncRNA gene-pairs: LSP1 and ENST00000509204.1 (A); HLA-DQB1 and ENST00000443574.1 (B); MST1 and ENST00000563780.1 (C); TSPAN33 and ENST00000498745.1 (D); SLC22A5 and ENST00000417795.1 (E); DGRD and ENST00000442524.1 (F). An extremely positive ( $r^{2} \geq 0.99$, P-value <2.2e-16) correlation was observed for MST1 and its intersecting IncRNA ENST00000563780.1, which is associated with IBD risk variants rs9822268 and rs3197999. Protein-coding expression is plotted on the $x$-axis, and IncRNA expression is shown on the $y$-axis. Each point represents a biopsy sample from different clinical subgroups.

expressed between disease and control. Conversely, the expression profile of 1,006 differentially expressed genes significantly associated with age $(P$-value $<0.05$, $t$-test; Table S10 in Additional file 1). These results suggest that even though the sample diagnosis for disease is only partly related to other clinical parameters, especially disease index and smoking, many differentially expressed genes in iCD and iUC also reflect an impact of the patient's age. The average gene significance measures for all genes in a given module are summarized in Table S10 in Additional file 1.

Overall, the network analysis identified three large coexpression modules enriched for differentially expressed genes between iCD/iUC and control $(P$-value $<10 \mathrm{e}-100$, Pearson's Chi-squared test; Figures S10 and S11 in Additional file 2). The three modules comprised 2,054 out of 2,737 differentially expressed genes. The gene network of the 'brown' module was found to be enriched for immune and pro-inflammatory responses (Table S11 in Additional file 1), the 'green' and 'red' modules were driven by genes involved in small molecule transmembrane transport, and anionic and cationic transport (Tables S12 and S13 in Additional file 1). GO analysis was also performed for the randomized gene sets of the same module sizes. None of the randomized modules had significant GO terms.

\section{Discussion}

The present study was intended to explore the transcriptomic landscape of lncRNAs in IBD, with particular focus on $\mathrm{CD}$ and $\mathrm{UC}$. To explore the transcriptomic profiles of $\mathrm{CD}$ and $\mathrm{UC}$ patients, colonic pinch biopsies were analyzed using gene expression microarrays. Our results revealed widespread dysregulation of lncRNA and protein-coding gene expression in both $\mathrm{CD}$ and $\mathrm{UC}$. It is noteworthy that although our main focus was transcriptome analysis of lncRNAs, we also profiled a significant number of protein-coding genes (approximately 12,000; see Methods). The Gencode v.15 lncRNA microarray has been extensively used and the levels of both mRNAs and lncRNAs are comparable and show strong correlations (ranging from 0.62 to 0.75 ) with results obtained from RNA sequencing (RNAseq) [38]. These correlations are also comparable with the previous lncRNA microarray versions [35]. The Gencode v.15 lncRNA microarray has been designed to capture both
poly(A) and non-poly(A) transcripts (out of a total 22,007 IncRNA transcripts targeted by the microarray, 9,273 lncRNA transcripts are polyadenylated). In recent years, many studies have been conducted to profile lncRNAs using RNAseq; however, this is expensive and time consuming because of the requirement of doing deep sequencing, particularly for lncRNAs, which are expressed at relatively lower levels than protein-coding genes [38]. It has also been reported that microarrays are more sensitive to detect whether a lncRNA is expressed or not compared with RNAseq [40].

SVM-based classifiers have been previously used to cross-validate the circulating microRNA-based biomarker panels in UC [13]. We also verified the robustness of the differentially expressed genes by SVM and the predictive capability of these genes to discriminate CD and UC was tested using SVM-RFE-based classifiers. The HarveyBradshaw Index and Simple Clinical Colitis Activity Index are symptom-based indices used to assess the disease activity in $\mathrm{CD}$ and $\mathrm{UC}$, respectively. Among various clinical parameters tested, we found strong influence of the disease index, followed by smoking, age and sex, on iCD and iUC phenotypes. Smoking is known to have deleterious effects in $C D$ while it has been found to be protective against UC [41]. Furthermore, smoking has also been shown to influence the colonic gene expression profile in CD [42]. Additionally, based on linear regression and WGCNA, we did not find any significant effect of biopsy location on overall gene expression. However, regional variation in gene expression along the colonic mucosa has been reported to influence expression profiling studies in IBD $[43,44]$. These modest regional variations are more pronounced in healthy controls and un-inflamed biopsies and largely remain masked when comparing inflamed biopsies [44]. On the contrary, other studies suggest no such gene expression differences due to regional variation $[17,45]$. These reports highlight the importance and impact of various confounding factors like smoking, sex, and biopsy locations among many other clinically relevant parameters in gene expression analysis in IBD.

Our analysis identified common expression patterns between the lncRNAs and protein-coding genes in iCD and iUC as confirmed by unsupervised hierarchical clustering (Figure 3). A distinctive inflammatory (iCD/iUC) gene expression signature included the key drivers of the innate and adaptive immune responses (chemokines, 

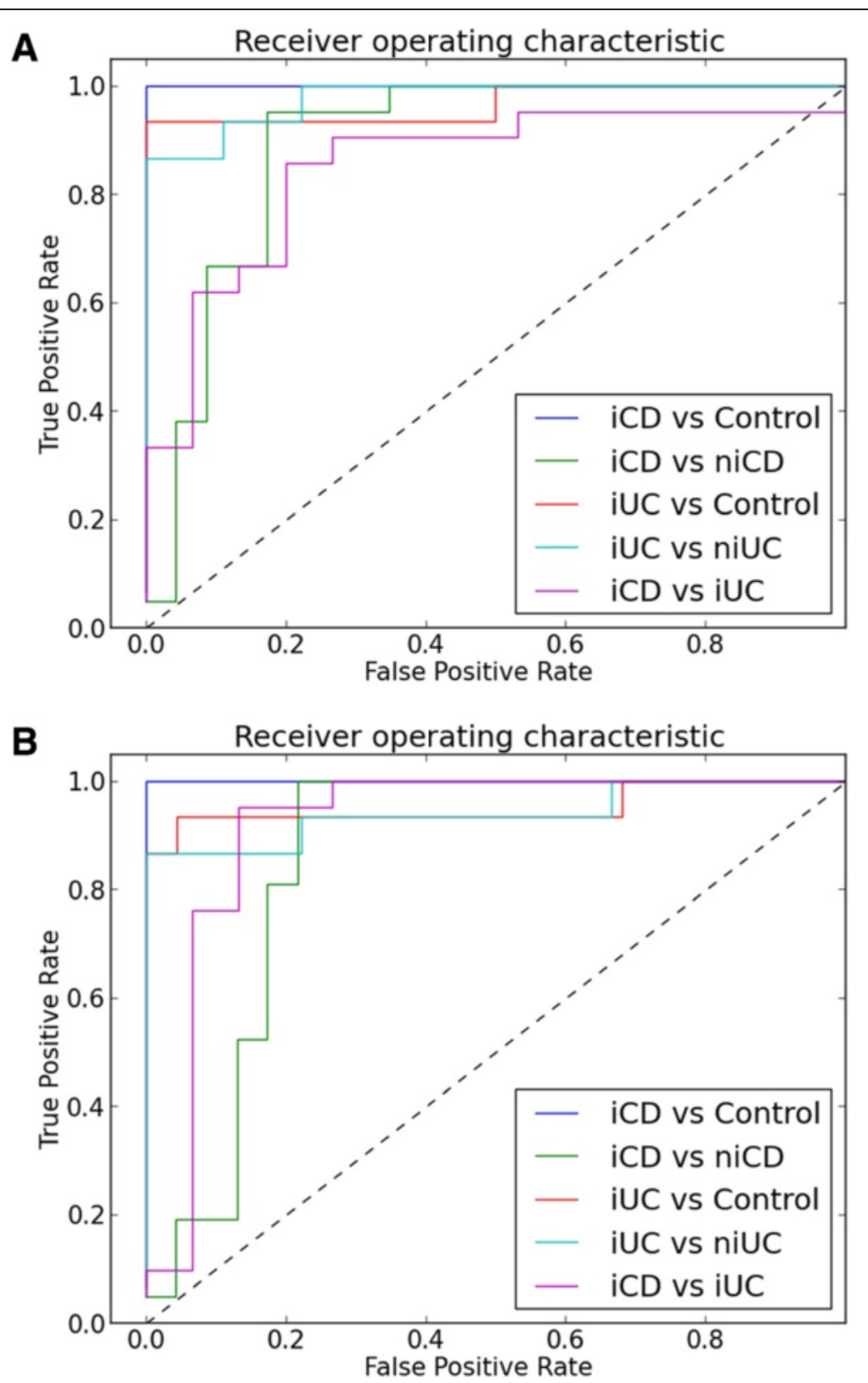

Figure 8 Receiver operating characteristic curve analysis of differentially expressed IncRNAs and protein-coding genes. (A) IncRNAs and (B) protein-coding genes for five comparisons.

cytokines and defensins) - for example, DUOXA2 (dual oxidase maturation factor 2), CXCL1 (chemokine (C-X-C motif) ligand 1), CXCL9 (chemokine (C-X-C motif) ligand 9) - and also included a significant number of lncRNAs. Expression levels of both DUOX2 and DUOXA2 have been reported to be up-regulated in association with iUC, and in UC-associated colorectal dysplasia and colorectal cancer and are involved specifically in inflammation and regulated on a crypt-by-crypt basis in UC [46]. We also observed a global up-regulation of DUOXA2 in iCD and iUC compared with both non- inflamed and healthy controls. Both DUOX2 and its maturation factor DUOXA2 are part of the NADPH oxidase family of enzymes involved in release of hydrogen peroxide $\left(\mathrm{H}_{2} \mathrm{O}_{2}\right)$ [47]. These enzymes are essential components of evolutionarily conserved mechanisms through which organisms are known to defend themselves against bacterial, viral, or parasitic infections, yet allowing tolerance of commensals [48,49] Suppression of DUOX2generated $\mathrm{H}_{2} \mathrm{O}_{2}$ production by mesalazine (5-aminosalicylic acid) has been demonstrated to reduce reactive oxygen species-induced genetic lesions and thereby lowering 
the risk of UC-associated colorectal dysplasia and colorectal cancer [46].

Our results revealed significant down-regulation of the lncRNA ANRIL (antisense non-coding RNA in the INK4 locus) in both $\mathrm{iCD}(\mathrm{FC}<-2.7, P$-value $<0.05)$ and iUC $($ FC $<-7.9, P$-value $<0.05)$ compared with non-inflamed and healthy controls. ANRIL, encoded on the chromosome 9p2.3 region, is a known hotspot for disease-associated SNPs [50]. ANRIL has emerged as an important regulatory molecule mediating human disease at various levels and cellular settings. Nevertheless, the role of ANRIL has not yet been described specifically in the context of IBD pathology. ANRIL has been found to be up-regulated in leukemia, prostate cancer, basal cell carcinoma and glioma, whereas depletion of ANRIL has been implicated in reduced proliferation, indicating its role in cancerogenesis [51-53]. Remarkably, in our study, eight major ANRIL isoforms, including the isoforms known to form circular variants (cANRIL), were found to be universally downregulated in both $\mathrm{iCD}$ and iUC. Importantly, endogenous expression of $c A N R I L$ has been associated with risk for atherosclerosis [54]. In this context, dysregulation of ANRIL in IBD is highly intriguing, particularly the downregulation of the $c A N R I L$ isoform. Indeed, recently, circular RNAs have been shown to be involved in stabilizing sense transcripts and also act as sponges for microRNAs [55]; however, the biological functions of circRNAs have recently been debated [56]. It is imperative, therefore, to investigate comprehensively the potential roles of $c A N R I L$ in IBD pathogenesis.

Unsurprisingly, our results also enabled us to distinguish between $\mathrm{iCD}$ and $\mathrm{IUC}$, although the number of differentially expressed genes was small, which emphasizes the close pathogenic nature of $\mathrm{CD}$ and UC. An interesting distinction between $\mathrm{iCD}$ and $\mathrm{iUC}$ involved the expression of SERPINB3 (serpin peptidase inhibitor, clade B (ovalbumin), member 3), which was significantly down-regulated $(\mathrm{FC}<-3.8)$ in iCD versus iUC (Table 6 and Figure 1G). SERPINB3 has been found to be overexpressed in certain squamous epithelial cancers, such as uterine cervix carcinoma, head and neck carcinomas, and esophagus carcinoma [57]. Although the precise physiological functions of SERPINB3 are elusive, it has been hypothesized that SERPINB3 might be involved in the development of autoimmunity [58].

In our study, we found significant enrichment for the 96 differentially expressed lncRNAs within IBD loci. Collectively, we found differentially expressed IBD lociassociated lncRNAs overlapping active regulatory elements within known binding motifs in intestinal epithelium and immune cells [7]. LncRNA RP3-395 M20.8 was found to be associated with the regulatory IBD risk variant rs10797432, which affects the binding motifs for $A P-2$ (transcription factor AP-2 alpha (activating enhancer binding protein 2 alpha)) and CTCF (CCCTC-binding factor (zinc finger protein)) (Table S5 in Additional file 1). Moreover, IBD risk variant rs1569723 is known to act as a cis-eQTL for CD40 (CD40 molecule, TNF receptor superfamily member 5), which was significantly up-regulated in iUC and associated with IncRNA RP11-465 L10.10. Additionally, lncRNA IFNG-ASI harboring UC susceptibility SNP rs7134599 was found to be up-regulated in iUC. SNP rs7134599 is associated with the IBD26 (12q15) genetic locus and with regulatory pro-inflammatory cytokines IFNG (interferon, gamma) and $I L-2$ (interleukin 2) and anti-inflammatory cytokine $I L-26$ (interleukin 26). IFNG gene encodes interferon gamma $(I F N-\gamma)$, a soluble cytokine that is pivotal for the host's innate and adaptive immunity against viral, certain bacterial and protozoal infections. Aberrant expression of $I F N-\gamma$ has been linked with a number of autoimmune and inflammatory diseases, and mucosal expression of IFN- $\gamma$ is known to play a vital role in the pathogenesis of IBD [59]. IL-2 is encoded by the IL2 gene and is involved in immune responses to microbial infections and intestinal inflammation activation in IBD. Anti-inflammatory $I L-26$ has been shown to be overexpressed in CD [60]. These findings suggest potential involvement of differentially expressed lncRNAs overlapping the active regulatory elements in IBD pathogenesis.

Interestingly, we also found positive $\left(r^{2} \geq 0.5\right)$ and extremely positive $\left(r^{2} \geq 0.9\right)$ correlations between the overlapping as well as cis-neighboring differentially expressed IBD loci-associated lncRNA-protein-coding gene pairs. A strong positive correlation was observed between lncRNA AC051649.12 and protein-coding gene LSP1 (lymphocyte-specific protein 1) associated with IBD risk variant rs907611. SNP rs907611 affects the binding affinity of transcriptional factors $Y Y 1$ and $N F-m u E 1$ and thus alters gene expression. It is plausible that the differentially expressed IBD loci-associated lncRNAs intersecting protein-coding genes somehow contribute to the regulation of the latter [61]. Taken together, these data suggest lncRNAs have a role in regulating the expression of IBD loci-associated genes.

Additionally, we also noticed dysregulation of AMPs and inflammatory response genes such as pro-inflammatory chemokines and cytokines in various clinical subgroups. For example, the key antimicrobial response genes REG3A (Regenerating islet-derived 3 alpha), DEFA5 (Defensin, alpha 5, Paneth cell-specific) and DEFA6 (Defensin, alpha 6 , Paneth cell-specific), were $>30$-fold up-regulated specially in $\mathrm{iCD}$ versus control. Consistent with our results, REG3A, DEFA5 and DEFA6 have been shown previously to be significantly up-regulated and linked to Paneth cell metaplasia in IBD $[62,63]$. Mutations in the cytoplasmic pathogen recognition receptor NOD2 (nucleotide-binding oligomerization domain containing 2) gene have been associated with ileal CD and Paneth cell dysfunction [64] 
and, importantly, NOD2 was found to be up-regulated in both iCD and iUC. Concordant with the findings by Arijs et al. [62], we also found two AMPs, DEFB1 and NPY, significantly down-regulated in both $\mathrm{ICD}$ and iUC. IL15 (interleukin 15) was found specifically up-regulated in $\mathrm{ICD}$ but not in iUC, which supports the notion that it contributes to acute intestinal inflammation in CD [65].

For all the differentially expressed lncRNAs and protein-coding genes, we evaluated biological functional processes through analysis of GO terms based on 'guiltby-association' and WGCNA. Unsurprisingly, we found enrichment for immune response, pro-inflammatory cytokine activity, extracellular matrix organization, and ion membrane transport genes (Tables S9, S11, S12 and S13 in Additional file 1). Given the idiopathic nature of IBD, the overall up-regulation of pro-inflammatory immune response-related gene expression could be largely due to the infiltrating immune cells, rather than the underlying disease phenotype. Indeed, persistent inflammation in $C D$ and UC is known to be elicited by the activation of innate and adaptive immune cells by foreign antigens, which in turn produce and release pro-inflammatory cytokines that give rise to the vicious circle of inflammation, thereby leading to chronic tissue injury and epithelial damage [66]. Nevertheless, differentially expressed genes identified in non-inflamed samples (niCD and niUC) versus control (Table S3 and S4 in Additional file 1) might be disease specific. In summary, our findings suggest that dysregulated lncRNAs could be involved in IBD pathogenesis. However, these findings warrant systematic experimental follow-up in cellular and murine models with additional validation in a larger cohort in order to elucidate the role and biomarker potential of these dysregulated lncRNAs in IBD.

\section{Conclusions}

We show that lncRNA expression profiling can be effectively used to stratify iCD and iUC from healthy controls. Additionally, our data indicate the underlying potential of IncRNA transcriptional signatures associated with clinical parameters as biomarkers for IBD.

\section{Additional files}

Additional file 1: Tables S1, S2, S3 and S4. The top differentially expressed IncRNAs and protein-coding genes for inflamed versus non-inflamed tissues in CD and UC. Table S1. Top 10 up/down-regulated IncRNAs and protein-coding genes in iCD versus niCD. Table S2. Top 10 up/down-regulated IncRNAs and protein-coding genes in iUC versus niUC. Table S3. Common differentially expressed genes found between iCD versus control and niCD versus control. Table S4. Common differentially expressed genes between iUC versus control and niUC versus control. Tables S5, S6 and S7. Table S5. List of oligonucleotides used for validating microarray data using real-time PCR analysis for selected 15 genes. Table S6. Validation of microarray results by real-time PCR analysis for eight differentially expressed genes in iCD versus control and iUC versus control. Table S7. Validation of microarray results by real-time PCR analysis for five differentially expressed genes in iCD versus iUC. Table S8: 96 differentially expressed IncRNAs found significantly enriched within IBD-loci. Table S9: Functional annotation of differentially expressed IncRNAs based on the nearest neighbor approach. Tables S10, S11, S12 and S13. Weighted correlation network analysis (WGCNA). Table S10. Gene significance. The co-expression network identified the gene significance for the different clinical parameters. Table S11. Brown module - immune and inflammatory response: 4,216 genes in the module, of which 1,748 have an Entrez ID (considered in GO analysis). Table S12. Green module - small molecule trans-membrane transport: 2,934 genes in the module, of which 1,210 have an Entrez ID (considered in GO analysis). Table S13. Red module - anionic and cationic transport: 2,486 genes in the module, of which 1,173 have an Entrez ID (considered in GO analysis).

Additional file 2: Figure S1. Pearson correlations calculated for the technical replicates (six samples analyzed in duplicates on separate chips: 16_2, 18_3, 27_2, 28_3, 47_3 and 21_2). Figure S2. Scatterplot matrices describing the variation explained by the first four principal components for 90 biopsy samples. Figure S3. Unsupervised hierarchical clustering of the most dynamic probes (coefficient of variance $>0.05$ ) targeting IncRNAs (S3A) and protein-coding genes (S3B) across the samples in different clinical subgroups. Figure S4. Log2 ratio and - $\log 10$ adjusted $P$-values plotted and represented as volcano plots for the non-inflamed tissue comparisons iCD versus niCD (S4A) and iUC versus niUC (S4B). Figure S5. Expression map of the top 40 differentially expressed IncRNAs and protein-coding genes in iCD versus iUC based on unsupervised hierarchical clustering. Figure S6. Expression map of the total differentially expressed IncRNAs and proteincoding genes in $\mathrm{C} C \mathrm{C}$ versus controls (S6A) and iUC versus controls (S6B) (patients in red, controls in blue) based on unsupervised hierarchical clustering. Figures S7, S8 and S9. Figure S7. Dendrogram of samples and heatmap of clinical parameters. Linear regression model and weighted correlation network analysis (WGCNA) were used to investigate the impact of clinical parameters on disease diagnosis. Figure S8. Receiver operating characteristic (ROC) curve analysis for age (a), sex (b), disease index (c), smoking (d) classification using differentially expressed IncRNAs in all five comparisons. Figure S9. Overlap of differentially expressed genes identified by LIMMA and SVM. Figures S10 and S11. Figure S10. Co-expression network is built by hierarchical clustering and Dynamic Tree Cut. Modules are clusters of highly interconnected genes. The 'brown', 'green' and 'red' modules are enriched for differentially expressed genes between iUC/iCD and control. Figure S11. Module significance is determined as the average absolute gene significance measure for all genes in a given module.

\section{Abbreviations}

AMP: antimicrobial peptide; CD: Crohn's disease; eQTL: expression quantitative trait locus; FC: fold change; FDR: false discovery rate; GO: Gene Ontology; IBD: inflammatory bowel disease; iCD: inflamed Crohn's disease; IFN: interferon; IL: interleukin; iUC: inflamed ulcerative colitis; IncRNA: long non-coding RNA; ncRNA: non-coding RNA; niCD: non-inflamed Crohn's disease; niUC: non-inflamed ulcerative colitis; PCA: principal component analysis; PCR: polymerase chain reaction; qPCR: quantitative PCR; RFE: recursive feature elimination; SNP: single-nucleotide polymorphism; SVM: Support Vector Machines; UC: ulcerative colitis; WGCNA: weighted correlation network analysis.

\section{Competing interests}

The authors declare that they have no competing interests.

\section{Authors' contributions}

Conceived and designed the experiments: FP, JG, CHBB, AHM, MV, KSF. Performed the experiments: AHM, CHBB, SES, XP. Analyzed the data and wrote the manuscript: AHM. Intellectual guidance: FP, JG. All authors read and approved the final manuscript.

\section{Acknowledgements}

We thank the Genomics Unit's microarray core facility at Centre for Genomic Regulation (CRG), Barcelona, Spain, for performing the microarray experiments. We thank Sarah Bonin and Rory Johnson for helpful advice for initial quality control of the data and Marc R Friedlander for help with sample logistics at CRG. We also would like to thank Lene Buhl Riis for histological evaluation of biopsies and Lene Normann Nielsen and Helle Buck Rasmussen for sample collection and handling. 


\section{Funding}

This work was supported by grants from Danish Council for Strategic Research, Danish Council for Independent Research (Technology and Production Sciences), Danish Center for Scientific Computing (DCSC, DeiC) and Poul and Erna Sehested Hansen Foundation for the funding. MV is supported by Dagny Sigismunde Marie Sofie Rasmine Hertz Johansens and Andreas Hermann Johansens Fond. XP is supported by Innovation Fund Denmark. The funders had no role in study design, data collection and analysis or preparation of the manuscript.

\section{Author details}

${ }^{1}$ Center for non-coding RNA in Technology and Health, University of Copenhagen, Frederiksberg 1870, Denmark. '2Department of Pediatrics E, Copenhagen Diabetes Research Center (CPH-DIRECT), Herlev University Hospital, Herlev 2730, Denmark. ${ }^{3}$ Faculty of Health and Medical Sciences, University of Copenhagen, Copenhagen 2200, Denmark. ${ }^{4}$ Department of Surgery, North Zealand Hospital, Hillerød 3400, Denmark. ${ }^{5}$ The Novo Nordisk Foundation Center for Protein Research, University of Copenhagen, Copenhagen 2200, Denmark. 'Department of Obesity Biology, Novo Nordisk, Måløv 2760, Denmark. ${ }^{7}$ Department of Molecular Genetics, Novo Nordisk, Måløv 2760, Denmark.

\section{Received: 28 November 2014 Accepted: 9 April 2015}

\section{Published online: 13 May 2015}

\section{References}

1. Van Limbergen J, Radford-Smith G, Satsangi J. Advances in IBD genetics. Nat Rev Gastroenterol Hepatol. 2014;11:372-85.

2. Xavier RJ, Podolsky DK. Unravelling the pathogenesis of inflammatory bowel disease. Nature. 2007;448:427-34.

3. Abraham C, Cho JH. Inflammatory bowel disease. N Engl J Med. 2009;361:2066-78.

4. Sartor RB. Genetics and environmental interactions shape the intestinal microbiome to promote inflammatory bowel disease versus mucosal homeostasis. Gastroenterology. 2010;139:1816-9.

5. Jostins L, Ripke S, Weersma RK, Duerr RH, McGovern DP, Hui KY, et al. Host-microbe interactions have shaped the genetic architecture of inflammatory bowel disease. Nature. 2012;491:119-24.

6. Mirza AH, Kaur S, Brorsson CA, Pociot F. Effects of GWAS-associated genetic variants on IncRNAs within IBD and T1D candidate loci. PLoS One 2014;9:e105723.

7. Mokry M, Middendorp S, Wiegerinck CL, Witte M, Teunissen H, Meddens CA, et al. Many inflammatory bowel disease risk loci include regions that regulate gene expression in immune cells and the intestinal epithelium. Gastroenterology. 2014;146:1040-7.

8. Pekow JR, Kwon JH. MicroRNAs in inflammatory bowel disease. Inflamm Bowel Dis. 2012;18:187-93.

9. Granlund A, Flatberg A, Østvik AE, Drozdov I, Gustafsson BI, Kidd M, et al Whole genome gene expression meta-analysis of inflammatory bowel disease colon mucosa demonstrates lack of major differences between Crohn's disease and ulcerative colitis. PLoS One. 2013;8:e56818.

10. Coskun $\mathrm{M}$, Bjerrum JT, Seidelin JB, Nielsen $\mathrm{OH}$. MicroRNAs in inflammatory bowel disease-pathogenesis, diagnostics and therapeutics. World J Gastroenterol. 2012;18:4629-34.

11. Lin J, Welker NC, Zhao Z, Li Y, Zhang J, Reuss SA, et al. Novel specific microRNA biomarkers in idiopathic inflammatory bowel disease unrelated to disease activity. Mod Pathol. 2014;27:602-8.

12. Iborra M, Bernuzzi F, Invernizzi P, Danese S. MicroRNAs in autoimmunity and inflammatory bowel disease: crucial regulators in immune response. Autoimmun Rev. 2012;11:305-14.

13. Duttagupta R, DiRienzo S, Jiang R, Bowers J, Gollub J, Kao J, et al. Genome-wide maps of circulating miRNA biomarkers for ulcerative colitis. PLoS One. 2012;7:e31241

14. Haberman Y, Tickle TL, Dexheimer PJ, Kim M-O, Tang D, Karns R, et al. Pediatric Crohn disease patients exhibit specific ileal transcriptome and microbiome signature. J Clin Invest. 2014;124:3617-33.

15. Brest $P$, Lapaquette $P$, Souidi $M$, Lebrigand K, Cesaro A, Vouret-Craviari V, et al. A synonymous variant in IRGM alters a binding site for miR-196 and causes deregulation of IRGM-dependent xenophagy in Crohn's disease. Nat Genet. 2011;43:242-5.
16. McKenna LB, Schug J, Vourekas A, McKenna JB, Bramswig NC, Friedman JR, et al. MicroRNAs control intestinal epithelial differentiation, architecture, and barrier function. Gastroenterology. 2010;139:1654-64.

17. Wu F, Dassopoulos T, Cope L, Maitra A, Brant SR, Harris ML, et al. Genome-wide gene expression differences in Crohn's disease and ulcerative colitis from endoscopic pinch biopsies: insights into distinctive pathogenesis. Inflamm Bowel Dis. 2007;13:807-21.

18. Olsen J, Gerds TA, Seidelin JB, Csillag C, Bjerrum JT, Troelsen JT, et al. Diagnosis of ulcerative colitis before onset of inflammation by multivariate modeling of genome-wide gene expression data. Inflamm Bowel Dis. 2009;15:1032-8.

19. Montero-Meléndez T, Llor X, García-Planella E, Perretti M, Suárez A Identification of novel predictor classifiers for inflammatory bowel disease by gene expression profiling. PLoS One. 2013;8:e76235.

20. Rapicavoli NA, Qu K, Zhang J, Mikhail M, Laberge R-M, Chang HY. A mammalian pseudogene IncRNA at the interface of inflammation and anti-inflammatory therapeutics. Elife. 2013;2:e00762.

21. Geboes K, Riddell R, Ost A, Jensfelt B, Persson T, Löfberg R. A reproducible grading scale for histological assessment of inflammation in ulcerative colitis. Gut. 2000;47:404-9.

22. D'Haens GR, Geboes K, Peeters M, Baert F, Penninckx F, Rutgeerts P. Early lesions of recurrent Crohn's disease caused by infusion of intestinal contents in excluded ileum. Gastroenterology. 1998;114:262-7.

23. GENCODE Custom IncRNA Expression Microarray Design. http://www. gencodegenes.org/Incrna_microarray.html. Accessed 2 August 2014.

24. Ritchie ME, Silver J, Oshlack A, Holmes M, Diyagama D, Holloway A, et al. A comparison of background correction methods for two-colour microarrays. Bioinformatics. 2007;23:2700-7.

25. Bolstad B. Probe Level Quantile Normalization of High Density Oligonucleotide Array Data. http://bmbolstad.com/stuff/qnorm.pdf. Accessed 20 October 2014

26. Smyth GK. Linear models and empirical bayes methods for assessing differential expression in microarray experiments. Stat Appl Genet Mol Biol. 2004;3:Article3.

27. Benjamini $Y$, Hochberg $Y$. Controlling the false discovery rate: a practical and powerful approach to multiple testing. J R Stat Soc B. 1995;57:289-300.

28. Gentleman RC, Carey VJ, Bates DM, Bolstad B, Dettling M, Dudoit S, et al. Bioconductor: open software development for computational biology and bioinformatics. Genome Biol. 2004;5:R80. www.bioconductor.org.

29. ImmunoBase. http://www.immunobase.org. Accessed 30 October 2014.

30. Quinlan AR, Hall IM. BEDTools: a flexible suite of utilities for comparing genomic features. Bioinformatics. 2010;26:841-2.

31. Boyle AP, Hong EL, Hariharan M, Cheng Y, Schaub MA, Kasowski M, et al. Annotation of functional variation in personal genomes using RegulomeDB. Genome Res. 2012;22:1790-7.

32. Mi H, Muruganujan A, Thomas PD. PANTHER in 2013: modeling the evolution of gene function, and other gene attributes, in the context of phylogenetic trees. Nucleic Acids Res. 2013;41:D377-86. http://www.pantherdb.org/.

33. Vapnik VN. The nature of statistical learning theory. 2nd edition. Statistics for engineering and information science. New York: Springer; 2000.

34. Pedregosa F, Varoquaux G, Gramfort A, Michel A, Thirion B, Grisel O, et al. Scikit-learn: machine learning in python. J Machine Learning Res. 2011;12:2825-30

35. Duan K-B, Rajapakse JC, Wang H, Azuaje F. Multiple SVM-RFE for gene selection in cancer classification with expression data. IEEE Trans Nanobioscience. 2005;4:228-34

36. Langfelder $P$, Horvath S. WGCNA: an R package for weighted correlation network analysis. BMC Bioinformatics. 2008;9:559.

37. Falcon S, Gentleman R. Using GOstats to test gene lists for GO term association. Bioinformatics. 2007;23:257-8.

38. Derrien T, Johnson R, Bussotti G, Tanzer A, Djebali S, Tilgner $H$, et al. The GENCODE v7 catalog of human long noncoding RNAs: analysis of their gene structure, evolution, and expression. Genome Res. 2012;22:1775-89.

39. Cabili MN, Trapnell C, Goff L, Koziol M, Tazon-Vega B, Regev A, et al. Integrative annotation of human large intergenic noncoding RNAs reveals global properties and specific subclasses. Genes Dev. 2011;25:1915-27.

40. Fu X, Fu N, Guo S, Yan Z, Xu Y, Hu H, et al. Estimating accuracy of RNA-Seq and microarrays with proteomics. BMC Genomics. 2009;10:161.

41. Khor B, Gardet A, Xavier RJ. Genetics and pathogenesis of inflammatory bowel disease. Nature. 2011;474:307-17. 
42. Nielsen OH, Bjerrum JT, Csillag C, Nielsen FC, Olsen J. Influence of smoking on colonic gene expression profile in Crohn's disease. PLoS One. 2009;4:e6210.

43. LaPointe LC, Dunne R, Brown GS, Worthley DL, Molloy PL, Wattchow D, et al. Map of differential transcript expression in the normal human large intestine. Physiol Genomics. 2008;33:50-64.

44. Noble CL, Abbas AR, Cornelius J, Lees CW, Ho G-T, Toy K, et al. Regional variation in gene expression in the healthy colon is dysregulated in ulcerative colitis. Gut. 2008;57:1398-405.

45. Costello CM, Mah N, Häsler R, Rosenstiel P, Waetzig GH, Hahn A, et al. Dissection of the inflammatory bowel disease transcriptome using genome-wide cDNA microarrays. PLoS Med. 2005;2:e199.

46. MacFie TS, Poulsom R, Parker A, Warnes G, Boitsova T, Nijhuis A, et al. DUOX2 and DUOXA2 form the predominant enzyme system capable of producing the reactive oxygen species $\mathrm{H} 2 \mathrm{O} 2$ in active ulcerative colitis and are modulated by 5-aminosalicylic acid. Inflamm Bowel Dis. 2014;20:514-24.

47. Bedard K, Krause K-H. The NOX family of ROS-generating NADPH oxidases: physiology and pathophysiology. Physiol Rev. 2007:87:245-313.

48. Bae YS, Choi MK, Lee W-J. Dual oxidase in mucosal immunity and host-microbe homeostasis. Trends Immunol. 2010;31:278-87.

49. Ha E-M, Lee K-A, Seo YY, Kim S-H, Lim J-H, Oh B-H, et al. Coordination of multiple dual oxidase-regulatory pathways in responses to commensal and infectious microbes in drosophila gut. Nat Immunol. 2009;10:949-57.

50. Pasmant $E$, Sabbagh A, Vidaud M, Bièche I. ANRIL, a long, noncoding RNA, is an unexpected major hotspot in GWAS. FASEB J. 2011;25:444-8.

51. Sherborne AL, Hosking FJ, Prasad RB, Kumar R, Koehler R, Vijayakrishnan J, et al. Variation in CDKN2A at 9p21.3 influences childhood acute lymphoblastic leukemia risk. Nat Genet. 2010:42:492-4.

52. Yap KL, Li S, Muñoz-Cabello AM, Raguz S, Zeng L, Mujtaba S, et al. Molecular interplay of the noncoding RNA ANRIL and methylated histone $\mathrm{H3}$ lysine 27 by polycomb CBX7 in transcriptional silencing of INK4a. Mol Cell. 2010;38:662-74.

53. Cunnington MS, Santibanez Koref M, Mayosi BM, Burn J, Keavney B. Chromosome 9p21 SNPs associated with multiple disease phenotypes correlate with ANRIL expression. PLoS Genet. 2010;6:e1000899.

54. Burd CE, Jeck WR, Liu Y, Sanoff HK, Wang Z, Sharpless NE. Expression of linear and novel circular forms of an INK4/ARF-associated non-coding RNA correlates with atherosclerosis risk. PLoS Genet. 2010;6:e1001233.

55. Hansen TB, Jensen TI, Clausen BH, Bramsen JB, Finsen B, Damgaard CK, et al. Natural RNA circles function as efficient microRNA sponges. Nature. 2013:495:384-8.

56. Guo JU, Agarwal V, Guo H, Bartel DP. Expanded identification and characterization of mammalian circular RNAs. Genome Biol. 2014;15:409.

57. Izuhara K, Ohta S, Kanaji S, Shiraishi H, Arima K. Recent progress in understanding the diversity of the human ov-serpin/clade B serpin family. Cell Mol Life Sci. 2008;65:2541-53.

58. Vidalino L, Doria A, Quarta S, Zen M, Gatta A, Pontisso P. SERPINB3, apoptosis and autoimmunity. Autoimmun Rev. 2009:9:108-12.

59. Neurath MF. Cytokines in inflammatory bowel disease. Nat Rev Immunol. 2014;14:329-42.

60. Dambacher J, Beigel F, Zitzmann K, De Toni EN, Göke B, Diepolder HM, et al. The role of the novel Th17 cytokine IL-26 in intestinal inflammation. Gut. 2009:58:1207-17.

61. Gingeras TR. Origin of phenotypes: genes and transcripts. Genome Res. 2007;17:682-90.

62. Arijs I, De Hertogh G, Lemaire K, Quintens R, Van Lommel L, Van Steen K, et al. Mucosal gene expression of antimicrobial peptides in inflammatory bowel disease before and after first infliximab treatment. PLoS One. 2009:4:e7984.

63. Wehkamp J, Schmid M, Stange EF. Defensins and other antimicrobial peptides in inflammatory bowel disease. Curr Opin Gastroenterol. 2007:23:370-8.

64. Hamm CM, Reimers MA, McCullough CK, Gorbe EB, Lu J, Gu CC, et al. NOD2 status and human ileal gene expression. Inflamm Bowel Dis. 2010;16:1649-57.

65. Schulthess J, Meresse B, Ramiro-Puig E, Montcuquet N, Darche S, Bègue B, et al. Interleukin-15-dependent NKp46+ innate lymphoid cells control intestinal inflammation by recruiting inflammatory monocytes. Immunity. 2012;37:108-21.

66. Bouma G, Strober W. The immunological and genetic basis of inflammatory bowel disease. Nat Rev Immunol. 2003·3:521-33.

\section{Submit your next manuscript to BioMed Central and take full advantage of:}

- Convenient online submission

- Thorough peer review

- No space constraints or color figure charges

- Immediate publication on acceptance

- Inclusion in PubMed, CAS, Scopus and Google Scholar

- Research which is freely available for redistribution

Submit your manuscript at www.biomedcentral.com/submit 